\title{
Outer Bounds for Multiple-Access Channels With Feedback Using Dependence Balance
}

\author{
Ravi Tandon, Student Member, IEEE, and Sennur Ulukus, Member, IEEE
}

\begin{abstract}
We use the idea of dependence balance to obtain a new outer bound for the capacity region of the discrete memoryless multiple-access channel with noiseless feedback (MAC-FB). We consider a binary additive noisy MAC-FB whose feedback capacity is not known. The binary additive noisy MAC considered in this paper can be viewed as the discrete counterpart of the Gaussian MAC-FB. Ozarow established that the capacity region of the two-user Gaussian MAC-FB is given by the cut-set bound. Our result shows that for the discrete version of the channel considered by Ozarow, this is not the case. Direct evaluation of our outer bound is intractable due to an involved auxiliary random variable whose large cardinality prohibits an exhaustive search. We overcome this difficulty by using a composite function and its properties to explicitly evaluate our outer bound. Our outer bound is strictly less than the cut-set bound at all points on the capacity region where feedback increases capacity. In addition, we explicitly evaluate the Cover-Leung achievable rate region for the binary additive noisy MAC-FB in consideration. Furthermore, using the tools developed for the evaluation of our outer bound, we also explicitly characterize the boundary of the feedback capacity region of the binary erasure MAC, for which the Cover-Leung achievable rate region is known to be tight. This last result confirms that the feedback strategies developed by Kramer for the binary erasure MAC are capacity achieving.
\end{abstract}

Index Terms-Cut-set bound, dependence balance, multiple-access channel with feedback (MAC-FB).

\section{INTRODUCTION}

$\mathbf{N}$ OISELESS feedback can increase the capacity region of the discrete memoryless multiple-access channel (MAC), unlike for the single-user discrete memoryless channel. This was shown by Gaarder and Wolf in [2] for the binary erasure MAC, which is defined as $Y=X_{1}+X_{2}$. Ozarow showed in [3] that feedback can also increase the capacity region of a two-user Gaussian MAC with feedback (MAC-FB). A constructive achievability scheme based on the classical Schalkwijk-Kailath [4] feedback scheme was shown to be optimal for the two-user Gaussian MAC-FB. Moreover, the cut-set outer bound was shown to be tight in this case.

Manuscript received May 29, 2008; revised May 13, 2009. Current version published September 23, 2009. This work was supported by the National Science Foundation (NSF) under Grants CCF 04-47613, CCF 05-14846, CNS 07-16311, and CCF 07-29127. The material in this paper was presented in part at the IEEE Global Communications Conference, New Orleans, LA, November 2008.

The authors are with the Department of Electrical and Computer Engineering, University of Maryland, College Park, MD 20742 USA (e-mail: ravit@umd. edu; ulukus@umd.edu).

Communicated by G. Kramer, Associate Editor for Shannon Theory.

Color versions of Figures 2-7 in this paper are available online at http://ieeexplore.ieee.org.

Digital Object Identifier 10.1109/TIT.2009.2027532
Subsequently, Cover and Leung obtained an achievable rate region for the general MAC-FB based on block Markov superposition coding [5]. Even though this region is in general larger than the capacity region of the MAC without feedback, it is not optimal for the two-user Gaussian MAC-FB, as was shown in [3]. Kramer [6] used the notion of directed information to obtain an expression for the capacity region of the discrete memoryless MAC-FB. Unfortunately, this expression is in an incomputable non-single-letter form. Recently, Bross and Lapidoth [7] proposed an achievable rate region for the two-user discrete memoryless MAC-FB and showed that their region includes the Cover-Leung region, the inclusion being strict for some channels.

For a specific class of MAC-FB, Willems [8] developed an outer bound that equals the Cover-Leung achievable rate region. For this class of MAC-FB, each channel input (say $X_{1}$ ) should be expressible as a deterministic function of the other channel input $\left(X_{2}\right)$ and the channel output $(Y)$. The binary erasure MAC considered by Gaarder and Wolf, where $Y=X_{1}+X_{2}$, falls into this class of channels. Therefore, Cover-Leung region is the feedback capacity region for the binary erasure MAC.

A general outer bound for MAC-FB is the cut-set bound. Although the cut-set bound was shown to be tight for the two-user Gaussian MAC-FB, it is in general loose. An intuitive reason for the cut-set bound to be loose for the general MAC-FB is its permissibility of arbitrary input distributions, some of which yielding rates which may not be achievable. For instance, even though Cover-Leung achievability scheme introduces correlation between $X_{1}$ and $X_{2}$, it is a limited form of correlation, as the channel inputs are conditionally independent given an auxiliary random variable, whereas the cut-set bound allows all possible correlations.

The idea of dependence balance was introduced by Hekstra and Willems in [9] to obtain an outer bound on the capacity region of the single-output two-way channel. The basic idea behind this outer bound is to restrict the set of allowable input distributions, consequently restricting arbitrary correlation between channel inputs. The authors also developed a parallel channel extension for the dependence balance bound. The parallel channel extension can be interpreted as follows: the parallel channel output can be considered as a genie-aided information which is made available at both transmitters and the receiver and it also affects the set of allowable input distributions through the dependence balance bound. Depending on the choice of the genie information (which is equivalent to choosing a parallel channel), there is an inherent tradeoff between the set of allowable input distributions and the excessive mutual information rate terms which appear in the rate expressions as a consequence 
of the parallel channel output. We will exploit this tradeoff provided by the parallel channel extension of the dependence balance bound to obtain a strict improvement over the cut-set bound for a particular MAC whose feedback capacity is not known.

To motivate the choice of our MAC, consider the binary erasure MAC used by Gaarder and Wolf given by $Y=X_{1}+X_{2}$. If we introduce binary additive noise at the channel output, then the channel becomes $Y=X_{1}+X_{2}+N$, where all $X_{1}, X_{2}$, and $N$ are binary and $N$ has a uniform distribution. This is a nondeterministic noisy MAC which does not fall into any class of channels for which the feedback capacity is known. We should mention that this particular MAC was extensively studied by Kramer in [6], [10], where the first improvement over the Cover-Leung achievable rate region was obtained.

We extend the idea of dependence balance to obtain an outer bound for the entire capacity region of this binary additive noisy MAC-FB. Direct evaluation of the parallel-channel-based dependence balance bound is intractable due to an involved auxiliary random variable whose large cardinality prohibits an exhaustive search. We use composite functions and their properties to obtain a simple characterization for our bound. Our outer bound strictly improves upon the cut-set bound at all points on the boundary where feedback increases capacity. In addition, we explicitly evaluate the Cover-Leung achievable rate region for our binary additive noisy MAC-FB.

We particularly focus on the symmetric-rate ${ }^{1}$ point on the feedback capacity region of this channel. Cover-Leung's achievable symmetric rate for this channel was obtained in [10] as $0.43621 \mathrm{bits} /$ transmission. In [10], Kramer obtained an improved symmetric-rate inner bound as 0.43879 bits/transmission by using superposition coding and binning with code trees. The cut-set upper bound on the symmetric rate was obtained in [10] as 0.45915 bits/transmission. We obtain a symmetric-rate upper bound of $0.45330 \mathrm{bits} /$ transmission which strictly improves upon the cut-set bound. Furthermore, we also show that a binary and uniform selection of the involved auxiliary random variable is sufficient to obtain our symmetric-rate upper bound.

It should be remarked that the channel we consider in this paper can be thought of as the discrete counterpart of the channel considered by Ozarow [3]. Although the cut-set bound was shown to be tight for the two-user Gaussian MAC-FB, our result shows that the cut-set bound is not tight for the discrete version of the additive noisy MAC-FB.

As an application of the properties of the composite functions developed in this paper, we are able to obtain the entire boundary of the capacity region of the binary erasure MAC-FB. The evaluation of the asymmetric rate pairs on the boundary of the feedback capacity region of the binary erasure MAC was mentioned as an open problem in [11]. It was shown in [12] that a binary and uniform auxiliary random variable $T$ is sufficient to attain the sum-rate point on the capacity region of the binary erasure MAC-FB. We show here that this is also the case for any asymmetric rate point on the boundary of the feedback capacity region. This result also complements the work of Kramer [13], where feedback strategies were developed for the binary erasure

\footnotetext{
${ }^{1}$ By symmetric-rate point, we refer to the maximum rate $R$ such that the rate
} pair $(R, R)$ lies in the capacity region of MAC-FB.

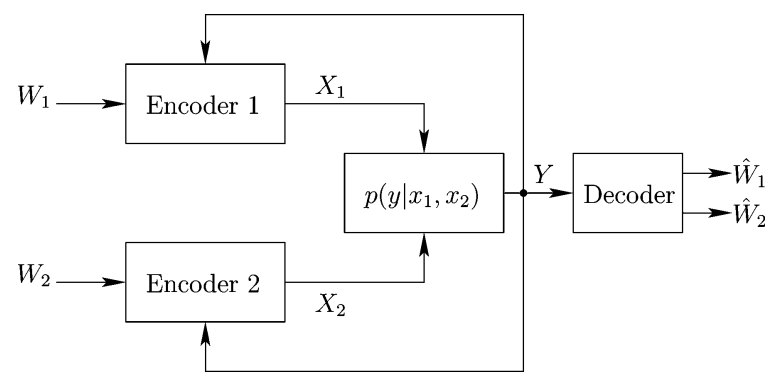

Fig. 1. The MAC with noiseless feedback (MAC-FB).

MAC-FB and it was shown that these strategies achieve all rates yielded by a binary selection of the auxiliary random variable $T$ in the capacity region. Our result hence shows in effect that the feedback strategies developed in [13] for binary erasure MAC are optimal and capacity achieving.

\section{SySTEM MODEL}

A discrete memoryless two-user MAC-FB (see Fig. 1) is defined by the following: two input alphabets $\mathcal{X}_{1}$ and $\mathcal{X}_{2}$, an output alphabet $\mathcal{Y}$, and the channel defined by a probability transition function $p\left(y \mid x_{1}, x_{2}\right)$ for all $\left(x_{1}, x_{2}, y\right) \in \mathcal{X}_{1} \times \mathcal{X}_{2} \times \mathcal{Y}$. An $\left(n, M_{1}, M_{2}, P_{e}\right)$ code for the MAC-FB consists of two sets of encoding functions $f_{1 i}, f_{2 i}$ for $i=1, \ldots, n$ and a decoding function $g$

$$
\begin{aligned}
& f_{1 i}: \mathcal{M}_{1} \times \mathcal{Y}^{i-1} \rightarrow \mathcal{X}_{1}, \quad i=1, \ldots, n \\
& f_{2 i}: \mathcal{M}_{2} \times \mathcal{Y}^{i-1} \rightarrow \mathcal{X}_{2}, \quad i=1, \ldots, n \\
& g: \mathcal{Y}^{n} \rightarrow \mathcal{M}_{1} \times \mathcal{M}_{2} .
\end{aligned}
$$

The two transmitters produce independent and uniformly distributed messages $W_{1} \in\left\{1, \ldots, M_{1}\right\}$ and $W_{2} \in\left\{1, \ldots, M_{2}\right\}$, respectively, and transmit them through $n$ channel uses. The average error probability is defined as $P_{e}=\operatorname{Pr}\left(g\left(Y^{n}\right) \neq\right.$ $\left.\left(W_{1}, W_{2}\right)\right)$. A rate pair $\left(R_{1}, R_{2}\right)$ is said to be achievable for MAC-FB if for any $\epsilon \geq 0$, there exists a pair of $n$ encoding functions $\left\{f_{1 i}\right\}_{i=1}^{n},\left\{f_{2 i}\right\}_{i=1}^{n}$, and a decoding function $g$ such that $R_{1} \leq \log \left(M_{1}\right) / n, R_{2} \leq \log \left(M_{2}\right) / n$, and $P_{e} \leq \epsilon$ for sufficiently large $n$. The capacity region of MAC-FB is the closure of the set of all achievable rate pairs $\left(R_{1}, R_{2}\right)$.

\section{Cut-Set Outer Bound FOR MAC-FB}

By applying Theorem 14.10.1 in [14], the cut-set outer bound on the capacity region of MAC-FB can be obtained as

$$
\begin{aligned}
\mathcal{C S}=\left\{\left(R_{1}, R_{2}\right):\right. & R_{1} \leq I\left(X_{1} ; Y \mid X_{2}\right) \\
& R_{2} \leq I\left(X_{2} ; Y \mid X_{1}\right) \\
& \left.R_{1}+R_{2} \leq I\left(X_{1}, X_{2} ; Y\right)\right\}
\end{aligned}
$$

where the random variables $\left(X_{1}, X_{2}, Y\right)$ have the joint distribution

$$
p\left(x_{1}, x_{2}, y\right)=p\left(x_{1}, x_{2}\right) p\left(y \mid x_{1}, x_{2}\right) .
$$

The cut-set outer bound allows all input distributions $p\left(x_{1}, x_{2}\right)$, which makes it seemingly loose since an achievable scheme 
might not achieve arbitrary correlation and rates given by the cut-set bound. Our aim is to restrict the set of allowable input distributions by using a dependence balance approach.

\section{DePendence BALANCE OUter Bound For MAC-FB}

Hekstra and Willems [9] showed that the capacity region of MAC-FB is contained within $\mathcal{D B}$, where

$$
\begin{aligned}
\mathcal{D B}=\left\{\left(R_{1}, R_{2}\right):\right. & R_{1} \leq I\left(X_{1} ; Y \mid X_{2}, T\right) \\
& R_{2} \leq I\left(X_{2} ; Y \mid X_{1}, T\right) \\
& \left.R_{1}+R_{2} \leq I\left(X_{1}, X_{2} ; Y \mid T\right)\right\}
\end{aligned}
$$

where the random variables $\left(X_{1}, X_{2}, Y, T\right)$ have the joint distribution

$$
p\left(t, x_{1}, x_{2}, y\right)=p(t) p\left(x_{1}, x_{2} \mid t\right) p\left(y \mid x_{1}, x_{2}\right)
$$

and also satisfy the following dependence balance bound:

$$
I\left(X_{1} ; X_{2} \mid T\right) \leq I\left(X_{1} ; X_{2} \mid Y, T\right)
$$

where $T$ is subject to a cardinality constraint of $|\mathcal{T}| \leq$ $\left|\mathcal{X}_{1}\right|\left|\mathcal{X}_{2}\right|+2$. The dependence balance bound restricts the set of input distributions in the sense that it allows only those input distributions $p\left(t, x_{1}, x_{2}\right)$ which satisfy (9). It should be noted that by ignoring the constraint in (9), one obtains the cut-set bound.

\section{AdAPtive Parallel Channel Extension of the DEPENDENCE BALANCE BOUND}

In [9], Hekstra and Willems also developed an adaptive parallel channel extension for the dependence balance bound which is given as follows: Let $\Delta(\mathcal{U})$ denote the set of all distributions of $U$ and $\Delta(\mathcal{U} \mid \mathcal{V})$ denote the set of all conditional distributions of $U$ given $V$. Then for any mapping $F: \Delta\left(\mathcal{X}_{1} \times\right.$ $\left.\mathcal{X}_{2}\right) \rightarrow \Delta\left(\mathcal{Z} \mid \mathcal{X}_{1} \times \mathcal{X}_{2} \times \mathcal{Y}\right)$, the capacity region of the MAC-FB is contained in

$$
\begin{aligned}
\mathcal{D B}_{P C}=\left\{\left(R_{1}, R_{2}\right):\right. & R_{1} \leq I\left(X_{1} ; Y, Z \mid X_{2}, T\right) \\
& R_{2} \leq I\left(X_{2} ; Y, Z \mid X_{1}, T\right) \\
& R_{1} \leq I\left(X_{1} ; Y \mid X_{2}\right) \\
& R_{2} \leq I\left(X_{2} ; Y \mid X_{1}\right) \\
& R_{1}+R_{2} \leq I\left(X_{1}, X_{2} ; Y\right) \\
& \left.R_{1}+R_{2} \leq I\left(X_{1}, X_{2} ; Y, Z \mid T\right)\right\}
\end{aligned}
$$

where the random variables $\left(X_{1}, X_{2}, Y, Z, T\right)$ have the joint distribution

$p\left(t, x_{1}, x_{2}, y, z\right)=p(t) p\left(x_{1}, x_{2} \mid t\right) p\left(y \mid x_{1} x_{2}\right) p^{+}\left(z \mid x_{1}, x_{2}, y, t\right)$

such that for all $t$

$$
p^{+}\left(z \mid x_{1}, x_{2}, y, t\right)=F\left(p_{X_{1} X_{2}}\left(x_{1}, x_{2} \mid t\right)\right)
$$

and such that

$$
I\left(X_{1} ; X_{2} \mid T\right) \leq I\left(X_{1} ; X_{2} \mid Y, Z, T\right)
$$

where $T$ is subject to a cardinality bound of $|\mathcal{T}| \leq\left|\mathcal{X}_{1}\right|\left|\mathcal{X}_{2}\right|+3$.
We should remark that the parallel channel (defined by $\left.p^{+}\left(z \mid x_{1}, x_{2}, y, t\right)\right)$ is selected a priori, and for every choice of the parallel channel, one obtains an outer bound on the capacity region of MAC-FB, which is in general tighter than the cut-set bound. The set of allowable input distributions $p\left(t, x_{1}, x_{2}\right)$ are those which satisfy the constraint in (18). Also note that only the right-hand side of (18), i.e., only $I\left(X_{1} ; X_{2} \mid Y, Z, T\right)$, depends on the choice of the parallel channel. By carefully selecting $p^{+}\left(z \mid x_{1}, x_{2}, y, t\right)$, one can reduce $I\left(X_{1} ; X_{2} \mid Y, Z, T\right)$, thereby making the constraint in (18) more stringent, consequently reducing the set of allowable input distributions. To obtain an improvement over the cut-set bound, we need to select a "good" parallel channel such that it restricts the input distributions to a small allowable set and yields small values of $I\left(X_{1} ; Z \mid Y, X_{2}, T\right)$ and $I\left(X_{2} ; Z \mid Y, X_{1}, T\right)$ at the same time. These two mutual information "leak" terms are the extra terms that appear in (10) and (11) relative to the rates appearing in (5) and (6), respectively.

To motivate the choice of our particular parallel channel, first consider a trivial choice of $Z: Z=\phi$ (a constant). For this choice of $Z,(18)$ reduces to (9) and we are not restricting the set of allowable input distributions any more than the $\mathcal{D B}$ bound. Moreover, for a constant selection of $Z,(10)$ and (11) reduce to (5) and (6), respectively. Thus, a constant selection of $Z$ for $\mathcal{D} \mathcal{B}_{P C}$ is equivalent to $\mathcal{D} \mathcal{B}$ itself.

Also note that the smallest value of $I\left(X_{1} ; X_{2} \mid Y, Z, T\right)$ is zero. Thus, it follows that if we select a parallel channel such that $I\left(X_{1} ; X_{2} \mid Y, Z, T\right)=0$ for every input distribution $p\left(t, x_{1}, x_{2}\right)$, then $I\left(X_{1} ; X_{2} \mid T\right)=0$ by (18). Hence, the smallest set of input distributions permissible by $\mathcal{D} \mathcal{B}_{P C}$ consists of those $p\left(t, x_{1}, x_{2}\right)$ for which $X_{1}$ and $X_{2}$ are conditionally independent given $T$. Furthermore, for a parallel channel such that $I\left(X_{1} ; X_{2} \mid Y, Z, T\right)=0$, the bound in (15) is redundant. This can be seen from

$$
\begin{aligned}
0= & I\left(X_{1} ; X_{2} \mid T\right)-I\left(X_{1} ; X_{2} \mid Y, Z, T\right) \\
= & I\left(X_{1} ; Y, Z \mid T\right)-I\left(X_{1} ; Y, Z \mid X_{2}, T\right) \\
= & I\left(X_{1}, X_{2} ; Y, Z \mid T\right)-I\left(X_{1} ; Y, Z \mid X_{2}, T\right) \\
& -I\left(X_{2} ; Y, Z \mid X_{1}, T\right) .
\end{aligned}
$$

Using (19), it is clear that the sum of constraints (10) and (11) is at least as strong as the constraint (15). This shows that $(15)$ is redundant for the class of parallel channels where $I\left(X_{1} ; X_{2} \mid Y, Z, T\right)=0$.

\section{BINARY ADDITIVE NOISY MAC-FB}

In this paper, we will consider a binary-input additive noisy MAC given by

$$
Y=X_{1}+X_{2}+N
$$

where $N$ is binary, uniform over $\{0,1\}$, and independent of $X_{1}$ and $X_{2}$. The channel output $Y$ takes values from the set $\mathcal{Y}=$ $\{0,1,2,3\}$. This channel does not fall into any class of MAC for which the feedback capacity region is known. This channel was also considered by Kramer in [6], [10] where it was shown that the Cover-Leung achievable rate is strictly suboptimal for the sum-rate. 
We select a parallel channel $p^{+}\left(z \mid x_{1}, x_{2}, y\right)$ such that $I\left(X_{1} ; X_{2} \mid Y, Z, T\right)=0$. By (18), this will imply $I\left(X_{1} ; X_{2} \mid T\right)=0$, and hence only distributions of the type

$$
p\left(t, x_{1}, x_{2}\right)=p(t) p\left(x_{1} \mid t\right) p\left(x_{2} \mid t\right)
$$

will be allowed. By doing so, we restrict the set of allowable input distributions to be the smallest permitted by $\mathcal{D} \mathcal{B}_{P C}$, although we pay a penalty due to the positive "leak" terms $I\left(X_{1} ; Z \mid Y, X_{2}, T\right)$ and $I\left(X_{2} ; Z \mid Y, X_{1}, T\right)$.

Two simple choices of $Z$ which yield $I\left(X_{1} ; X_{2} \mid Y, Z, T\right)=$ 0 are $Z=X_{1}$ and $Z=X_{2}$. For each of these choices, the corresponding outer bounds are

$$
\begin{aligned}
\mathcal{D} \mathcal{B}_{P C}^{(1)}=\left\{\left(R_{1}, R_{2}\right):\right. & R_{1} \leq I\left(X_{1} ; Y \mid X_{2}, T\right) \\
& +H\left(X_{1} \mid Y, X_{2}, T\right) \\
R_{2} \leq & I\left(X_{2} ; Y \mid X_{1}, T\right) \\
R_{1} \leq & I\left(X_{1} ; Y \mid X_{2}\right) \\
R_{1} & \left.+R_{2} \leq I\left(X_{1}, X_{2} ; Y\right)\right\}
\end{aligned}
$$

and

$$
\begin{aligned}
\mathcal{D B} & (2)=\left\{\left(R_{1}, R_{2}\right):\right. \\
R_{1} \leq & I\left(X_{1} ; Y \mid X_{2}, T\right) \\
R_{2} \leq & I\left(X_{2} ; Y \mid X_{1}, T\right) \\
& +H\left(X_{2} \mid Y, X_{1}, T\right) \\
R_{2} \leq & I\left(X_{2} ; Y \mid X_{1}\right) \\
R_{1} & \left.+R_{2} \leq I\left(X_{1}, X_{2} ; Y\right)\right\}
\end{aligned}
$$

where both $\mathcal{D} \mathcal{B}_{P C}^{(1)}$ and $\mathcal{D} \mathcal{B}_{P C}^{(2)}$ are evaluated over the set of input distributions of the form $p\left(t, x_{1}, x_{2}\right)=p(t) p\left(x_{1} \mid t\right) p\left(x_{2} \mid t\right)$. We should remark here that these two outer bounds can also be obtained by extending the approach of Zhang, Berger, and Schalkwijk [15] to the MAC-FB.

Lemma 1: For the binary additive noisy MAC-FB given in (20), the following equalities hold for any distribution of the form $p\left(t, x_{1}, x_{2}\right)=p(t) p\left(x_{1} \mid t\right) p\left(x_{2} \mid t\right)$ :

$$
\begin{aligned}
H\left(X_{1} \mid Y, X_{2}, T\right) & =\frac{1}{2} H\left(X_{1} \mid T\right) \\
H\left(X_{2} \mid Y, X_{1}, T\right) & =\frac{1}{2} H\left(X_{2} \mid T\right) .
\end{aligned}
$$

The proof of Lemma 1 is given in the Appendix .

Using Lemma 1, we can simplify $\mathcal{D} \mathcal{B}_{P C}^{(1)}$ and $\mathcal{D} \mathcal{B}_{P C}^{(2)}$ as

$$
\mathcal{D} \mathcal{B}_{P C}^{(1)}=\left\{\left(R_{1}, R_{2}\right): R_{1} \leq \min \left(I\left(X_{1} ; Y \mid X_{2}\right), H\left(X_{1} \mid T\right)\right)\right.
$$

$$
\begin{aligned}
& R_{2} \leq \frac{1}{2} H\left(X_{2} \mid T\right) \\
& \left.R_{1}+R_{2} \leq I\left(X_{1}, X_{2} ; Y\right)\right\}
\end{aligned}
$$

and

$$
\begin{aligned}
\mathcal{D B} \mathcal{B}_{P C}^{(2)}=\left\{\left(R_{1}, R_{2}\right):\right. & R_{1} \leq \frac{1}{2} H\left(X_{1} \mid T\right) \\
R_{2} & \leq \min \left(I\left(X_{2} ; Y \mid X_{1}\right), H\left(X_{2} \mid T\right)\right) \\
& \left.R_{1}+R_{2} \leq I\left(X_{1}, X_{2} ; Y\right)\right\}
\end{aligned}
$$

where both bounds are evaluated over the set of distributions of the form $p\left(t, x_{1}, x_{2}\right)=p(t) p\left(x_{1} \mid t\right) p\left(x_{2} \mid t\right)$ and the auxiliary random variable $T$ is subject to a cardinality constraint of $|\mathcal{T}| \leq\left|\mathcal{X}_{1}\right|\left|\mathcal{X}_{2}\right|+3$. The evaluation of the above outer bounds is rather cumbersome because for binary inputs, the bound on $|\mathcal{T}|$ is $|\mathcal{T}| \leq 7$. To the best of our knowledge, no one has been able to conduct an exhaustive search over an auxiliary random variable whose cardinality is larger than 4 . In Section VIII, we will obtain an alternate characterization for our outer bounds using composite functions and their properties. For that, we will first develop some useful properties of composite functions in the next section.

A valid outer bound is given by the intersection of $\mathcal{D B}(1)$ and $\mathcal{D B}_{P C}^{(2)}$ :

$$
\mathcal{D B}_{P C}=\mathcal{D B} \mathcal{B}_{P C}^{(1)} \bigcap \mathcal{D} \mathcal{B}_{P C}^{(2)} \text {. }
$$

We will show that this outer bound is strictly smaller than the cut-set bound at all points on the capacity region where feedback increases capacity.

\section{COMPosite Functions AND Their PROPERTIES}

Before obtaining a characterization of our outer bounds, we will define a composite function and prove two lemmas regarding its properties. These lemmas will be essential in obtaining simple characterizations for our outer bounds and the Cover-Leung achievable rate region. Throughout the paper, we will refer to the entropy function as $h^{(k)}\left(s_{1}, \ldots, s_{k}\right)$ which is defined as

$$
h^{(k)}\left(s_{1}, \ldots, s_{k}\right)=-\sum_{i=1}^{k} s_{i} \log \left(s_{i}\right)
$$

for $s_{i} \geq 0, i=1 \ldots, k$, and $\sum_{i=1}^{k} s_{i}=1$, where all logarithms are to the base 2 . We will denote $h^{(2)}(s, 1-s)$ simply as $h(s)$. To characterize our bounds, we will make use of the following function:

$$
\phi(s)= \begin{cases}\frac{1-\sqrt{1-2 s}}{2}, & \text { for } 0 \leq s \leq 1 / 2 \\ \frac{1-\sqrt{2 s-1}}{2}, & \text { for } 1 / 2<s \leq 1 .\end{cases}
$$

It was shown in [12] that the composite function $h(\phi(s))$ is symmetric around $s=1 / 2$ and concave in $s$ for $0 \leq s \leq 1$. The functions $\phi(s)$ and $h(\phi(s))$ are illustrated in Fig. 2. From the definition of $\phi(s)$ in (39) it is clear that for any $s \in[0,1]$, the function $\phi(s)$ satisfies the following property:

$$
\phi(2 s(1-s))=\min (s, 1-s) .
$$

As a consequence, the following holds as well:

$$
h(\phi(2 s(1-s)))=h(s) .
$$

For any $s \in[0,1]$, the following holds from the definition of $\phi(s)$ :

$$
s= \begin{cases}\phi(2 s(1-s)), & 0 \leq s \leq \frac{1}{2} \\ 1-\phi(2 s(1-s)), & \frac{1}{2}<s \leq 1 .\end{cases}
$$




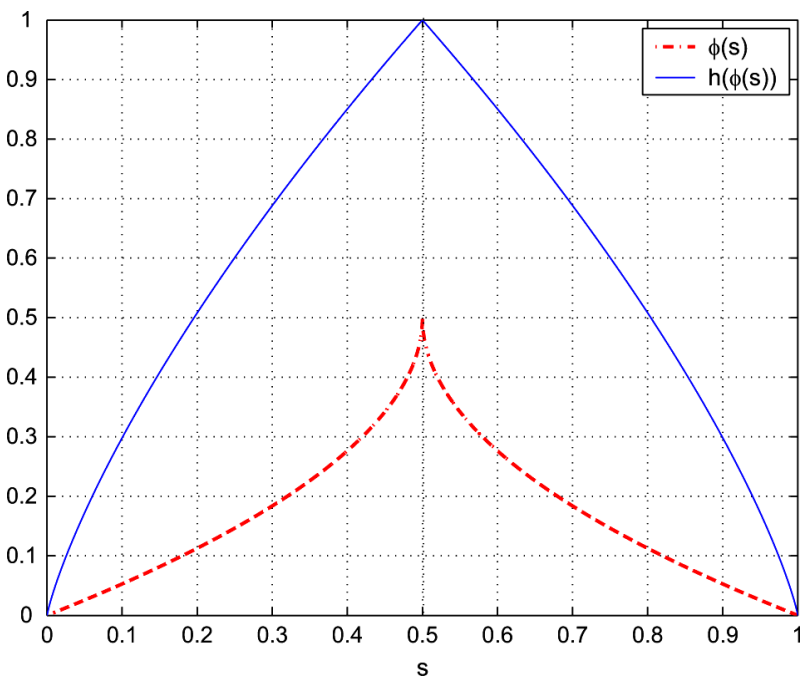

Fig. 2. Functions $\phi(s)$ and $h(\phi(s))$.

For any $x \in\left[0, \frac{1}{2}\right]$ and $y \in\left[0, \frac{1}{2}\right]$, let us define a function

$$
\begin{aligned}
f(x, y) & \triangleq \phi(x)+\phi(y)-2 \phi(x) \phi(y) \\
& =\frac{1-\sqrt{(1-2 x)(1-2 y)}}{2} .
\end{aligned}
$$

From the above definition, it is clear that the function $f(x, y)$ lies in the range $[0,1 / 2]$. We now state two lemmas regarding the function $f(x, y)$.

Lemma 2: The variable

$$
v=s_{1}+s_{2}-2 s_{1} s_{2}
$$

is always lower-bounded by $f\left(2 s_{1}\left(1-s_{1}\right), 2 s_{2}\left(1-s_{2}\right)\right)$ for any $s_{1} \in[0,1], s_{2} \in[0,1]$.

Lemma 3: The function $f(x, y)$ is jointly convex in $(x, y)$ for $0 \leq x \leq \frac{1}{2}, 0 \leq y \leq \frac{1}{2}$.

The proofs of Lemmas 2 and 3 are given in the Appendix.

\section{EVAluAtion of THE DePENDENCE BALANCE OUteR BOUND}

We now present the main result of this paper.

Theorem 1: The feedback capacity region of the binary additive noisy MAC given by (20) is contained in the region

$$
\mathcal{D} \mathcal{B}_{P C}=\mathcal{D} \mathcal{B}_{P C}^{(1)} \bigcap \mathcal{D} \mathcal{B}_{P C}^{(2)}
$$

where

$$
\begin{aligned}
\mathcal{D B} & (1)=\bigcup_{\left(u_{1}, u_{2}, u\right) \in \mathcal{P}}\left\{\left(R_{1}, R_{2}\right):\right. \\
R_{1} & \leq \min \left(\frac{1}{2} h(u), h\left(\phi\left(2 u_{1}\right)\right)\right) \\
R_{2} & \leq \frac{1}{2} h\left(\phi\left(2 u_{2}\right)\right) \\
R_{1} & \left.+R_{2} \leq h\left(\frac{1-u}{2}\right)\right\}
\end{aligned}
$$

and

$$
\begin{aligned}
\mathcal{D B} & (2)=\bigcup_{\left(u_{1}, u_{2}, u\right) \in \mathcal{P}}\left\{\left(R_{1}, R_{2}\right):\right. \\
R_{1} & \leq \frac{1}{2} h\left(\phi\left(2 u_{1}\right)\right) \\
R_{2} & \leq \min \left(\frac{1}{2} h(u), h\left(\phi\left(2 u_{2}\right)\right)\right) \\
R_{1} & \left.+R_{2} \leq h\left(\frac{1-u}{2}\right)\right\}
\end{aligned}
$$

and the set $\mathcal{P}$ is defined as

$$
\begin{gathered}
\mathcal{P}=\left\{\left(u_{1}, u_{2}, u\right): 0 \leq u_{1} \leq \frac{1}{4} ; 0 \leq u_{2} \leq \frac{1}{4} ;\right. \\
\left.f\left(2 u_{1}, 2 u_{2}\right) \leq u \leq 1-\left(u_{1}+u_{2}\right)\right\}
\end{gathered}
$$

Proof: We will explicitly characterize our outer bounds $\mathcal{D} \mathcal{B}_{P C}^{(1)}$ and $\mathcal{D} \mathcal{B}_{P C}^{(2)}$. Let the cardinality of the auxiliary random variable $T$ be fixed and arbitrary, say $|\mathcal{T}|$. Then, the joint distribution $p(t) p\left(x_{1} \mid t\right) p\left(x_{2} \mid t\right)$ can be described by the following variables:

$$
\begin{aligned}
q_{1 t}=\operatorname{Pr}\left(X_{1}=0 \mid T=t\right), & & t=1, \ldots,|\mathcal{T}| \\
q_{2 t}=\operatorname{Pr}\left(X_{2}=0 \mid T=t\right), & & t=1, \ldots,|\mathcal{T}| \\
p_{t}=\operatorname{Pr}(T=t), & & t=1, \ldots,|\mathcal{T}| .
\end{aligned}
$$

We will characterize our outer bounds in terms of three variables $u_{1}, u_{2}$, and $u$ which are functions of $p\left(t, x_{1}, x_{2}\right)$, and are defined as

$$
\begin{aligned}
u_{1} & =\sum_{t} p_{t} q_{1 t}\left(1-q_{1 t}\right)=\sum_{t} p_{t} u_{1 t} \\
u_{2} & =\sum_{t} p_{t} q_{2 t}\left(1-q_{2 t}\right)=\sum_{t} p_{t} u_{2 t} \\
u & =\sum_{t} p_{t}\left(q_{1 t}+q_{2 t}-2 q_{1 t} q_{2 t}\right)=\sum_{t} p_{t} u_{t}
\end{aligned}
$$

where we have defined

$$
\begin{aligned}
u_{1 t} & =q_{1 t}\left(1-q_{1 t}\right) \\
u_{2 t} & =q_{2 t}\left(1-q_{2 t}\right) \\
u_{t} & =q_{1 t}+q_{2 t}-2 q_{1 t} q_{2 t} .
\end{aligned}
$$

It should be noted that since $0 \leq q_{j t} \leq 1$, for $j=1,2, t=$ $1, \ldots,|\mathcal{T}|$, the variables $u_{1}, u_{2}, u_{1 t}$, and $u_{2 t}$ all lie in the range $\left[0, \frac{1}{4}\right]$. Our outer bounds $\mathcal{D B}_{P C}^{(1)}$ and $\mathcal{D} \mathcal{B}_{P C}^{(2)}$ are comprised of the following information-theoretic entities:

1) $\quad H\left(X_{1} \mid T\right), H\left(X_{2} \mid T\right)$;

2) $I\left(X_{1} ; Y \mid X_{2}\right), I\left(X_{2} ; Y \mid X_{1}\right)$;

3) $I\left(X_{1}, X_{2} ; Y\right)$.

We will first obtain upper bounds for each one of these entities individually in terms of $\left(u_{1}, u_{2}, u\right)$.

We upper-bound $H\left(X_{1} \mid T\right)$ as follows:

$$
\begin{aligned}
H\left(X_{1} \mid T\right) & =\sum_{t} p_{t} h\left(q_{1 t}\right) \\
& =\sum_{t} p_{t} h\left(\phi\left(2 q_{1 t}\left(1-q_{1 t}\right)\right)\right)
\end{aligned}
$$




$$
\begin{aligned}
& =\sum_{t} p_{t} h\left(\phi\left(2 u_{1 t}\right)\right) \\
& \leq h\left(\phi\left(2 u_{1}\right)\right)
\end{aligned}
$$

where (58) follows due to (41), (59) follows from (54), and (60) follows from the fact that $h(\phi(s))$ is concave in $s$ and the application of Jensen's inequality [14]. Using a similar set of inequalities for $H\left(X_{2} \mid T\right)$, we obtain

$$
H\left(X_{2} \mid T\right) \leq h\left(\phi\left(2 u_{2}\right)\right) .
$$

We will now upper-bound $I\left(X_{1} ; Y \mid X_{2}\right)$ in terms of the variable $u$. For this purpose, let us first define

$$
\begin{aligned}
a & =P_{X_{1} X_{2}}(0,0)=\sum_{t} p_{t} q_{1 t} q_{2 t} \\
b & =P_{X_{1} X_{2}}(0,1)=\sum_{t} p_{t} q_{1 t}\left(1-q_{2 t}\right) \\
c & =P_{X_{1} X_{2}}(1,0)=\sum_{t} p_{t}\left(1-q_{1 t}\right) q_{2 t} \\
d & =P_{X_{1} X_{2}}(1,1)=1-a-b-c .
\end{aligned}
$$

We now proceed as

$$
\begin{aligned}
I\left(X_{1} ; Y \mid X_{2}\right)= & H\left(Y \mid X_{2}\right)-H\left(Y \mid X_{1}, X_{2}\right) \\
= & H\left(Y \mid X_{2}\right)-1 \\
= & (a+c) h^{(3)}\left(\frac{a}{2(a+c)}, \frac{1}{2}, \frac{c}{2(a+c)}\right) \\
& +(b+d) h^{(3)}\left(\frac{b}{2(b+d)}, \frac{1}{2}, \frac{d}{2(b+d)}\right)-1 \\
\leq & h^{(3)}\left(\frac{a+d}{2}, \frac{1}{2}, \frac{b+c}{2}\right)-1 \\
= & \frac{1}{2} h(b+c) \\
= & \frac{1}{2} h(u)
\end{aligned}
$$

where (69) follows by the concavity of the entropy function and the application of Jensen's inequality [14]. Using a similar set of inequalities, we also have

$$
I\left(X_{2} ; Y \mid X_{1}\right) \leq \frac{1}{2} h(u)
$$

We will now obtain an upper bound on $I\left(X_{1}, X_{2} ; Y\right)$. First note that

$$
\begin{aligned}
I\left(X_{1}, X_{2} ; Y\right) & =H(Y)-H\left(Y \mid X_{1}, X_{2}\right) \\
& =h^{(4)}\left(P_{Y}(0), P_{Y}(1), P_{Y}(2), P_{Y}(3)\right)-1
\end{aligned}
$$

where

$$
\begin{aligned}
& P_{Y}(0)=\sum_{t} p_{t} q_{1 t} q_{2 t} / 2 \\
& P_{Y}(1)=\sum_{t} p_{t}\left(q_{1 t}+q_{2 t}-q_{1 t} q_{2 t}\right) / 2 \\
& P_{Y}(2)=\sum_{t} p_{t}\left(1-q_{1 t} q_{2 t}\right) / 2 \\
& P_{Y}(3)=\sum_{t} p_{t}\left(1-q_{1 t}\right)\left(1-q_{2 t}\right) / 2 .
\end{aligned}
$$

Using the following fact:

$$
\begin{aligned}
h^{(4)}(\alpha, \beta, \gamma, \theta) & =\frac{1}{2} h^{(4)}(\alpha, \beta, \gamma, \theta)+\frac{1}{2} h^{(4)}(\theta, \gamma, \beta, \alpha) \\
& \leq h^{(4)}\left(\frac{\alpha+\theta}{2}, \frac{\beta+\gamma}{2}, \frac{\beta+\gamma}{2}, \frac{\alpha+\theta}{2}\right) \\
& =h(\alpha+\theta)+h\left(\frac{1}{2}\right) \\
& =h(1-(\beta+\gamma))+1
\end{aligned}
$$

where (80) follows by the concavity of the entropy function and the application of Jensen's inequality [14], we now obtain an upper bound on $I\left(X_{1}, X_{2} ; Y\right)$ by continuing from (74)

$$
\begin{aligned}
I\left(X_{1}, X_{2} ; Y\right) & =h^{(4)}\left(P_{Y}(0), P_{Y}(1), P_{Y}(2), P_{Y}(3)\right)-1 \\
& \leq h\left(1-\left(P_{Y}(1)+P_{Y}(2)\right)\right)+h\left(\frac{1}{2}\right)-1 \\
& =h\left(\frac{1-u}{2}\right)
\end{aligned}
$$

where (84) follows by (82) and (85) follows from the fact that $P_{Y}(1)+P_{Y}(2)=(1+u) / 2$ using (76) and (77), where $u$ is as defined in (53).

We have obtained upper bounds on the information-theoretic entities which comprise our outer bounds in terms of three variables $u_{1}, u_{2}$, and $u$. We will now give a feasible region for these triples based on the structures of these variables. We claim that the following set is feasible:

$$
\begin{gathered}
\mathcal{P}=\left\{\left(u_{1}, u_{2}, u\right): 0 \leq u_{1} \leq \frac{1}{4} ; 0 \leq u_{2} \leq \frac{1}{4} ;\right. \\
\left.f\left(2 u_{1}, 2 u_{2}\right) \leq u \leq 1-\left(u_{1}+u_{2}\right)\right\} .
\end{gathered}
$$

First, note that for any $q_{1 t} \in[0,1]$, the following holds: $u_{1 t}=$ $q_{1 t}\left(1-q_{1 t}\right) \leq \frac{1}{4}$. Similarly, $u_{2 t}=q_{2 t}\left(1-q_{2 t}\right) \leq \frac{1}{4}$. Hence, we have

$$
\begin{aligned}
& 0 \leq u_{1} \leq \frac{1}{4} \\
& 0 \leq u_{2} \leq \frac{1}{4} .
\end{aligned}
$$

We now obtain a lower bound on $u$ as

$$
\begin{aligned}
u & =\sum_{t} p_{t} u_{t} \\
& \geq \sum_{t} p_{t} f\left(2 u_{1 t}, 2 u_{2 t}\right) \\
& \geq f\left(2 \sum_{t} p_{t} u_{1 t}, 2 \sum_{t} p_{t} u_{2 t}\right) \\
& =f\left(2 u_{1}, 2 u_{2}\right)
\end{aligned}
$$

where (90) follows by Lemma 2 and (91) follows by Lemma 3 and the application of Jensen's inequality [14]. We now obtain another lower bound on $u$

$$
\begin{aligned}
u & =\sum_{t} p_{t} u_{t} \\
& =\sum_{t} p_{t}\left(q_{1 t}+q_{2 t}-2 q_{1 t} q_{2 t}\right)
\end{aligned}
$$




$$
\begin{aligned}
& =\sum_{t} p_{t}\left(q_{1 t}-q_{1 t}^{2}+q_{2 t}-q_{2 t}^{2}+\left(q_{1 t}-q_{2 t}\right)^{2}\right) \\
& \geq \sum_{t} p_{t}\left(q_{1 t}-q_{1 t}^{2}+q_{2 t}-q_{2 t}^{2}\right) \\
& =\sum_{t} p_{t} q_{1 t}\left(1-q_{1 t}\right)+\sum_{t} p_{t} q_{2 t}\left(1-q_{2 t}\right) \\
& =u_{1}+u_{2}
\end{aligned}
$$

Finally, we obtain an upper bound on $u$ in terms of $u_{1}$ and $u_{2}$

$$
\begin{aligned}
u= & \sum_{t} p_{t} u_{t} \\
= & \sum_{t} p_{t}\left(q_{1 t}+q_{2 t}-2 q_{1 t} q_{2 t}\right) \\
= & \sum_{t} p_{t}\left(q_{1 t}+q_{2 t}-2 q_{1 t} q_{2 t}+q_{1 t}^{2}\right. \\
& \left.+\left(1-q_{2 t}\right)^{2}-q_{1 t}^{2}-\left(1-q_{2 t}\right)^{2}\right) \\
\leq & \sum_{t} p_{t}\left(q_{1 t}+q_{2 t}-2 q_{1 t} q_{2 t}+q_{1 t}^{2}\right. \\
& \left.+\left(1-q_{2 t}\right)^{2}-2 q_{1 t}\left(1-q_{2 t}\right)\right) \\
= & 1-\left(u_{1}+u_{2}\right)
\end{aligned}
$$

where (102) follows by the inequality $q_{1 t}^{2}+\left(1-q_{2 t}\right)^{2} \geq 2 q_{1 t}(1-$ $\left.q_{2 t}\right)$.

By noting that

$$
\begin{aligned}
f & \left.2 u_{1}, 2 u_{2}\right)-\left(u_{1}+u_{2}\right) \\
& =\frac{1-\sqrt{\left(1-4 u_{1}\right)\left(1-4 u_{2}\right)}}{2}-\left(u_{1}+u_{2}\right) \\
& =\frac{\left(1-4 u_{1}\right)+\left(1-4 u_{2}\right)-2 \sqrt{\left(1-4 u_{1}\right)\left(1-4 u_{2}\right)}}{4} \\
& =\frac{\left(\sqrt{1-4 u_{1}}-\sqrt{1-4 u_{2}}\right)^{2}}{4} \\
& \geq 0
\end{aligned}
$$

and using (92), we note that the lower bound in (98) is redundant.

Therefore, from (92) and (103), we have the following feasible range for the variable $u$ in terms of $u_{1}$ and $u_{2}$ :

$$
f\left(2 u_{1}, 2 u_{2}\right) \leq u \leq 1-\left(u_{1}+u_{2}\right) .
$$

Combining (87), (88), and (108), we obtain the set of feasible $\left(u_{1}, u_{2}, u\right)$ given in (86).

From (107), observe that $f\left(2 u_{1}, 2 u_{2}\right)=u_{1}+u_{2}$ only if $u_{1}=u_{2}$. The lower bound $u_{1}+u_{2} \leq u$ was sufficient for characterizing the symmetric feedback capacity of the binary erasure MAC [12]. Moreover, this characterization was possible by using only one variable $u$. On the other hand, our outer bounds are asymmetric in terms of the expressions appearing in the individual upper bounds for $R_{1}$ and $R_{2}$. Therefore, the bifurcation of information contained in any input distribution $p(t) p\left(x_{1} \mid t\right) p\left(x_{2} \mid t\right)$ in terms of three variables $\left(u_{1}, u_{2}, u\right)$ and an improved lower bound ${ }^{2}$ on the variable $u$ using the nonlinear

\footnotetext{
${ }^{2}$ Note that from the definition of $u$ in (53), we also have $u=\operatorname{Pr}\left(X_{1} \neq X_{2}\right)$. Therefore, roughly speaking, $1-u$ reflects the correlation between $X_{1}$ and $X_{2}$. Hence, obtaining a good lower bound on $u$ is equivalent to limiting the correlation between $X_{1}$ and $X_{2}$. This interpretation is in accordance with the basic idea of dependence balance.
}

bivariate function $f\left(2 u_{1}, 2 u_{2}\right)$ turn out to be crucial in capturing this asymmetry. This can be considered as a heuristic explanation as to why we are able to obtain explicit characterizations of our outer bounds and the Cover-Leung achievable rate region for both channel models considered in this paper.

It should be noted that the set of triples $\mathcal{P}$ obtained in (86) may not necessarily be the smallest feasible set of all triples $\left(u_{1}, u_{2}, u\right)$. Since we are interested in a maximization over these set of triples, a possibly larger set $\mathcal{P}$ suffices.

Using the upper bounds on $H\left(X_{1} \mid T\right), H\left(X_{2} \mid T\right)$, $I\left(X_{1} ; Y \mid X_{2}\right), I\left(X_{2} ; Y \mid X_{1}\right)$, and $I\left(X_{1}, X_{2} ; Y\right)$ in (60), (61), (71), (72), and (85) in terms of $\left(u_{1}, u_{2}, u\right)$ along with a feasible set of triples $\mathcal{P}$ in (86), we arrive at the desired characterizations for $\mathcal{D} \mathcal{B}_{P C}^{(1)}$ and $\mathcal{D} \mathcal{B}_{P C}^{(2)}$ given in (47) and (48), respectively. Finally, the intersection of two outer bounds is also a valid outer bound. Therefore, the outer bound $\mathcal{D} \mathcal{B}_{P C}$ given in (46) contains the feedback capacity region of the binary additive noisy MAC.

We will plot these outer bounds and their intersection in Fig. 4. In the next section, we will explicitly characterize our upper bounds for the symmetric-rate point on the feedback capacity region of the binary additive noisy MAC.

\section{EXPlicit ChaRACTERIZATION OF THE SyMmETRIC-RATE UPPER BOUND}

For the binary additive noisy MAC-FB in consideration, it was shown by Kramer [6] that the symmetric-rate cut-set bound is 0.45915 bits/transmission. It was also shown in [6] that the Cover-Leung achievable symmetric rate is 0.43621 bits/transmission and it was improved to 0.43879 bits/transmission by using superposition coding and binning with code trees. For completeness and comparison with existing bounds, we will first completely characterize our outer bound for the symmetric rate by providing the input distribution $p(t) p\left(x_{1} \mid t\right) p\left(x_{2} \mid t\right)$ which achieves it. By symmetric rate we mean a rate $R$ such that the rate pair $(R, R)$ lies in the capacity region of MAC-FB. For the symmetric rate, both $\mathcal{D} \mathcal{B}_{P C}^{(1)}$ and $\mathcal{D B} \mathcal{B}_{P C}^{(2)}$ will yield the same upper bound. Hence, we will focus on $\mathcal{D} \mathcal{B}_{P C}^{(1)}$. Using (47), we are interested in obtaining the largest $R$ over all $\left(u_{1}, u_{2}, u\right) \in \mathcal{P}$ such that

$$
\begin{aligned}
R & \leq \min \left(\frac{1}{2} h(u), h\left(\phi\left(2 u_{1}\right)\right)\right) \\
R & \leq \frac{1}{2} h\left(\phi\left(2 u_{2}\right)\right) \\
2 R & \leq h\left(\frac{1-u}{2}\right) .
\end{aligned}
$$

We will show that a seemingly weaker version of the above bound will improve upon the symmetric-rate cut-set bound. We will also show that the weaker bound is in fact the same as the above bound, and its sole purpose is the simplicity of evaluation and insight into the input distribution that attains it. We first obtain a weakened version of (109) as

$$
R \leq \min \left(\frac{1}{2} h(u), h\left(\phi\left(2 u_{1}\right)\right)\right) \leq h\left(\phi\left(2 u_{1}\right)\right) .
$$

Next, consider (111)

$$
2 R \leq h\left(\frac{1-u}{2}\right)
$$




$$
\begin{aligned}
& =h\left(\frac{1}{2}-\frac{u}{2}\right) \\
& \leq h\left(\frac{1}{2}-\frac{f\left(2 u_{1}, 2 u_{2}\right)}{2}\right)
\end{aligned}
$$

where (115) follows from (92) and the fact that the binary entropy function $h(s)$ is monotonically increasing in $s$ for $s \in$ $\left[0, \frac{1}{2}\right]$. Combining (110), (112), and (115), we are interested in the largest $R$ such that

$$
\begin{aligned}
R \leq & \max _{u_{1}, u_{2} \in\left[0, \frac{1}{4}\right]} \min \left(h\left(\phi\left(2 u_{1}\right)\right), \frac{1}{2} h\left(\phi\left(2 u_{2}\right)\right),\right. \\
& \left.\frac{1}{2} h\left(\frac{1}{2}-\frac{f\left(2 u_{1}, 2 u_{2}\right)}{2}\right)\right) .
\end{aligned}
$$

We note that this upper bound on the symmetric rate depends only on $u_{1}$ and $u_{2}$, and therefore, we replace the feasible set $\mathcal{P}$ with $u_{1}, u_{2} \in\left[0, \frac{1}{4}\right]$.

We know that $h(\phi(s))$ is concave in $s$ for $s \in[0,1]$. Hence, it follows that both $h\left(\phi\left(2 u_{1}\right)\right)$ and $\frac{1}{2} h\left(\phi\left(2 u_{2}\right)\right)$ are concave in $u_{1}$ and $u_{2}$, respectively, and hence concave in the pair $\left(u_{1}, u_{2}\right)$. We also have the following lemma.

Lemma 4: The function

$$
g\left(u_{1}, u_{2}\right)=\frac{1}{2} h\left(\frac{1-f\left(2 u_{1}, 2 u_{2}\right)}{2}\right)
$$

is monotonically decreasing and jointly concave in the pair $\left(u_{1}, u_{2}\right)$ for $u_{1}, u_{2} \in\left[0, \frac{1}{4}\right]$.

The proof of Lemma 4 is given in the Appendix .

Using Lemma 4, we conclude that all three functions in the $\min (\cdot)$ in $(116)$ are concave in $\left(u_{1}, u_{2}\right)$. Invoking the fact that the minimum of concave functions is concave, we conclude that the maximum in (116) is unique. We will now show that the unique pair $\left(u_{1}^{*}, u_{2}^{*}\right)$ that attains this maximum satisfies the property that $h\left(\phi\left(2 u_{1}^{*}\right)\right)=\frac{1}{2} h\left(\phi\left(2 u_{2}^{*}\right)\right)=g\left(u_{1}^{*}, u_{2}^{*}\right)$.

For this purpose, we first characterize those pairs $\left(\tilde{u}_{1}, \tilde{u}_{2}\right)$ such that the following holds:

$$
h\left(\phi\left(2 \tilde{u}_{1}\right)\right)=\frac{1}{2} h\left(\phi\left(2 \tilde{u}_{2}\right)\right)=g\left(\tilde{u}_{1}, \tilde{u}_{2}\right) .
$$

By using (118), we obtain two equations for $\tilde{u}_{1}$ and $\tilde{u}_{2}$, as

$$
\begin{aligned}
h\left(\phi\left(2 \tilde{u}_{1}\right)\right) & =\frac{1}{2} h\left(\frac{1-\phi\left(2 \tilde{u}_{1}\right)}{3-2 \phi\left(2 \tilde{u}_{1}\right)}\right) \\
\phi\left(2 \tilde{u}_{2}\right) & =\frac{1-\phi\left(2 \tilde{u}_{1}\right)}{3-2 \phi\left(2 \tilde{u}_{1}\right)} .
\end{aligned}
$$

From (119), one can see that $2 \tilde{u}_{1}$ is the unique solution $s \in$ $\left[0, \frac{1}{2}\right]$ of the equation

$$
h(\phi(s))=\frac{1}{2} h\left(\frac{1-\phi(s)}{3-2 \phi(s)}\right) .
$$

Obtaining the optimal $\tilde{u}_{1}$ from the above equation is illustrated in Fig. 3. The unique solutions $\left(\tilde{u}_{1}, \tilde{u}_{2}\right)$ of (119) and (120) are

$$
\tilde{u}_{1}=0.086063, \quad \tilde{u}_{2}=0.218333 \text {. }
$$

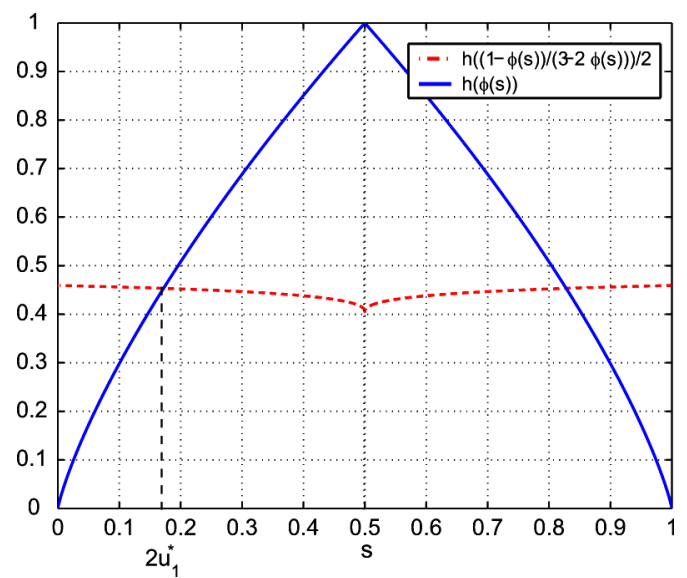

Fig. 3. Characterization of the optimal $u_{1}^{*}$.

We will now show that this pair $\left(\tilde{u}_{1}, \tilde{u}_{2}\right)$ yields the maximum in (116)

Returning to the maximization problem (116), first denote $\mathcal{S}$ as the region of allowable $\left(u_{1}, u_{2}\right)$

$$
\mathcal{S}=\left\{\left(u_{1}, u_{2}\right): 0 \leq u_{1} \leq \frac{1}{4} ; 0 \leq u_{2} \leq \frac{1}{4}\right\} .
$$

Also, define a subset of this region

$$
\tilde{\mathcal{S}}=\left\{\left(u_{1}, u_{2}\right): u_{1} \in\left(\tilde{u}_{1}, \frac{1}{4}\right) ; u_{2} \in\left(\tilde{u}_{2}, \frac{1}{4}\right)\right\}
$$

where $\left(\tilde{u}_{1}, \tilde{u}_{2}\right)$ is given by (122). We will now show that the pair $\left(\tilde{u}_{1}, \tilde{u}_{2}\right)$ yields the solution of the maximization problem in (116). Consider the following two cases.

1) If $\left(u_{1}, u_{2}\right) \in \tilde{\mathcal{S}}$, then by Lemma 4 , we have that $g\left(u_{1}, u_{2}\right) \leq g\left(\tilde{u}_{1}, \tilde{u}_{2}\right)$, using which we obtain

$$
\begin{aligned}
\min \left(h\left(\phi\left(2 u_{1}\right)\right), \frac{1}{2}\right. & \left.h\left(\phi\left(2 u_{2}\right)\right), g\left(u_{1}, u_{2}\right)\right) \\
& \leq g\left(u_{1}, u_{2}\right) \leq g\left(\tilde{u}_{1}, \tilde{u}_{2}\right) .
\end{aligned}
$$

2) If $\left(u_{1}, u_{2}\right) \in \mathcal{S} \backslash \tilde{\mathcal{S}}$, we either have $u_{1} \leq \tilde{u}_{1}$ or $u_{2} \leq \tilde{u}_{2}$ or both. Using this along with the fact that $h(\phi(2 s))$ is monotonically increasing in $s$ for $s \in\left[0, \frac{1}{4}\right]$, we obtain

$$
\min \left(h\left(\phi\left(2 u_{1}\right)\right), \frac{1}{2} h\left(\phi\left(2 u_{2}\right)\right), g\left(u_{1}, u_{2}\right)\right) \leq h\left(\phi\left(2 \tilde{u}_{1}\right)\right) .
$$

The above two cases show the following:

$$
\begin{aligned}
\max _{u_{1} \in\left[0, \frac{1}{4}\right], u_{2} \in\left[0, \frac{1}{4}\right]} \min \left(h\left(\phi\left(2 u_{1}\right)\right)\right. & \left., \frac{1}{2} h\left(\phi\left(2 u_{2}\right)\right), g\left(u_{1}, u_{2}\right)\right) \\
& =h\left(\phi\left(2 \tilde{u}_{1}\right)\right) \\
& =\frac{1}{2} h\left(\phi\left(2 \tilde{u}_{2}\right)\right) \\
& =g\left(\tilde{u}_{1}, \tilde{u}_{2}\right) .
\end{aligned}
$$

Thus, the maximum in (116) is obtained at $\left(u_{1}^{*}, u_{2}^{*}\right)=\left(\tilde{u}_{1}, \tilde{u}_{2}\right)$. We now obtain a distribution $p(t) p\left(x_{1} \mid t\right) p\left(x_{2} \mid t\right)$ which attains this symmetric-rate upper bound. Fix $T$ to be binary, and select the involved probabilities as

$$
\begin{aligned}
p_{0} & =p_{1}=\frac{1}{2} \\
q_{10} & =1-q_{11}=\phi\left(2 u_{1}^{*}\right) \\
q_{20} & =1-q_{21}=\phi\left(2 u_{2}^{*}\right) .
\end{aligned}
$$


The reason for constructing such an input distribution is that, at this specific distribution, we have the following exact equalities:

$$
\begin{aligned}
H\left(X_{1} \mid T\right) & =h\left(\phi\left(2 u_{1}^{*}\right)\right) \\
\frac{1}{2} H\left(X_{2} \mid T\right) & =\frac{1}{2} h\left(\phi\left(2 u_{2}^{*}\right)\right) \\
\frac{1}{2} I\left(X_{1}, X_{2} ; Y\right) & =g\left(u_{1}^{*}, u_{2}^{*}\right)
\end{aligned}
$$

and we achieve the outer bound we developed with equality. Substituting the values of $\left(u_{1}^{*}, u_{2}^{*}\right)$, we obtain a distribution given by

$$
\begin{aligned}
p_{0} & =p_{1}=\frac{1}{2} \\
q_{10} & =1-q_{11}=0.095109 \\
q_{20} & =1-q_{21}=0.322050 .
\end{aligned}
$$

The above input distribution yields a symmetric rate of $0.45330 \mathrm{bits} /$ transmission. Moreover, the $u^{*}$ corresponding to this distribution is given by

$$
\begin{aligned}
u^{*} & =\sum_{t} p_{t}\left(q_{1 t}+q_{2 t}-2 q_{1 t} q_{2 t}\right) \\
& =f\left(2 u_{1}^{*}, 2 u_{2}^{*}\right) \\
& =0.355899
\end{aligned}
$$

where (140) is by construction of the input distribution $p\left(t, x_{1}, x_{2}\right)$ and (141) is obtained by substituting the distribution specified in (136)-(138). Moreover, $\phi\left(2 u_{2}^{*}\right)<u^{*}<\frac{1}{2}$, hence, we also have that

$$
\frac{1}{2} h\left(u^{*}\right) \geq \frac{1}{2} h\left(\phi\left(2 u_{2}^{*}\right)\right)=h\left(\phi\left(2 u_{1}^{*}\right)\right) .
$$

This shows that the weakened version of the upper bound obtained in (116) is indeed tight and a binary auxiliary random variable $T$ with uniform distribution over $\{0,1\}$ is sufficient to attain this symmetric-rate upper bound.

\section{Evaluation of the Cover-Leung Achievable Rate REGION}

For completeness we will also obtain a simple characterization of the Cover-Leung inner bound for our binary additive noisy MAC-FB. For this purpose, we follow a two-step approach. In the first step, we first obtain an outer bound on the achievable rate region in terms of two variables $\left(u_{1}, u_{2}\right)$. In the second step, we specify an input distribution, as a function of $\left(u_{1}, u_{2}\right)$, which achieves the outer bound. We therefore arrive at an alternate characterization of the Cover-Leung achievable rate region in terms of the variables $\left(u_{1}, u_{2}\right)$.

The Cover-Leung achievable rate region [5] is given as

$$
\begin{aligned}
\mathcal{C} \mathcal{L}=\left\{\left(R_{1}, R_{2}\right)\right. & : R_{1} \leq I\left(X_{1} ; Y \mid X_{2}, T\right) \\
& R_{2} \leq I\left(X_{2} ; Y \mid X_{1}, T\right) \\
& \left.R_{1}+R_{2} \leq I\left(X_{1}, X_{2} ; Y\right)\right\}
\end{aligned}
$$

where the random variables $\left(T, X_{1}, X_{2}, Y\right)$ have the joint distribution

$$
p\left(t, x_{1}, x_{2}, y\right)=p(t) p\left(x_{1} \mid t\right) p\left(x_{2} \mid t\right) p\left(y \mid x_{1}, x_{2}\right)
$$

and the random variable $T$ is subject to a cardinality constraint of $|\mathcal{T}| \leq \min \left(\left|\mathcal{X}_{1}\right|\left|\mathcal{X}_{2}\right|+1,|\mathcal{Y}|+2\right)$.

We now state the main result of this section.

Theorem 2: The Cover-Leung achievable rate region for the binary additive noisy MAC given by (20) is given as

$$
\begin{aligned}
& \mathcal{C} \mathcal{L}=\bigcup_{\left(u_{1}, u_{2}\right) \in \mathcal{S}}\left\{\left(R_{1}, R_{2}\right)\right.: R_{1} \leq \frac{1}{2} h\left(\phi\left(2 u_{1}\right)\right) \\
& R_{2} \leq \frac{1}{2} h\left(\phi\left(2 u_{2}\right)\right) \\
&\left.R_{1}+R_{2} \leq h\left(\frac{1-f\left(2 u_{1}, u_{2}\right)}{2}\right)\right\}
\end{aligned}
$$

where the set $\mathcal{S}$ is defined as

$$
\mathcal{S}=\left\{\left(u_{1}, u_{2}\right): 0 \leq u_{1} \leq \frac{1}{4} ; 0 \leq u_{2} \leq \frac{1}{4}\right\} .
$$

Proof: For the binary additive noisy MAC in consideration, the constraints in (143)-(145) simplify as

$$
\begin{aligned}
R_{1} & \leq \frac{1}{2} H\left(X_{1} \mid T\right) \\
R_{2} & \leq \frac{1}{2} H\left(X_{2} \mid T\right) \\
R_{1}+R_{2} & \leq I\left(X_{1}, X_{2} ; Y\right) .
\end{aligned}
$$

We will first obtain an outer bound on the region specified by (149)-(151) in terms of two variables $\left(u_{1}, u_{2}\right)$. For every pair $\left(u_{1}, u_{2}\right)$, we will then specify an input distribution which will attain this outer bound. Note that the three constraints (149)-(151) are of similar form as in the case of $\mathcal{D} \mathcal{B}_{P C}^{(1)}$ and $\mathcal{D} \mathcal{B}_{P C}^{(2)}$, and we proceed in a similar manner to obtain upper bounds on the three terms above in terms of $u_{1}$ and $u_{2}$ as

$$
\begin{aligned}
R_{1} & \leq \frac{1}{2} h\left(\phi\left(2 u_{1}\right)\right) \\
R_{2} & \leq \frac{1}{2} h\left(\phi\left(2 u_{2}\right)\right) \\
R_{1}+R_{2} & \leq h\left(\frac{1-f\left(2 u_{1}, u_{2}\right)}{2}\right)
\end{aligned}
$$

where the variables $\left(u_{1}, u_{2}\right)$ belong to the set $\mathcal{S}$ defined in (123). Hence, an outer bound on the rate region specified by (149)-(151) is given as $\mathcal{O}$, where

$$
\begin{aligned}
& \mathcal{O}=\bigcup_{\left(u_{1}, u_{2}\right) \in \mathcal{S}}\left\{\left(R_{1}, R_{2}\right): R_{1} \leq \frac{1}{2} h\left(\phi\left(2 u_{1}\right)\right)\right. \\
& R_{2} \leq \frac{1}{2} h\left(\phi\left(2 u_{2}\right)\right) \\
& \left.R_{1}+R_{2} \leq h\left(\frac{1-f\left(2 u_{1}, u_{2}\right)}{2}\right)\right\} \text {. }
\end{aligned}
$$


Let $\left(u_{1}, u_{2}\right)$ be any arbitrary pair which belongs to $\mathcal{S}$. Consider an input distribution for which $|\mathcal{T}|=2$, and $T$ is uniform over $\{0,1\}$ and

$$
\begin{aligned}
p_{0} & =p_{1}=\frac{1}{2} \\
q_{10} & =1-q_{11}=\phi\left(2 u_{1}\right) \\
q_{20} & =1-q_{21}=\phi\left(2 u_{2}\right) .
\end{aligned}
$$

For this input distribution, we obtain the following exact equalities:

$$
\begin{aligned}
H\left(X_{1} \mid T\right) & =h\left(\phi\left(2 u_{1}\right)\right) \\
H\left(X_{2} \mid T\right) & =h\left(\phi\left(2 u_{2}\right)\right) \\
I\left(X_{1}, X_{2} ; Y\right) & =h\left(\frac{1-f\left(2 u_{1}, 2 u_{2}\right)}{2}\right) .
\end{aligned}
$$

We have thus shown that the outer bound we obtained on the achievable rate region in terms of $\left(u_{1}, u_{2}\right)$ can be attained by a set of input distributions for which the involved auxiliary random variable $T$ is binary and uniform. This in turn implies that a binary and uniform random variable $T$ is sufficient to characterize the entire Cover-Leung achievable rate region for the binary additive noisy MAC-FB. By varying over all such input distributions, or equivalently, by varying $\left(u_{1}, u_{2}\right)$ in the set $\mathcal{S}$, we obtain the entire Cover-Leung achievable rate region given in (147).

We should remark here that when evaluating the $\mathcal{D B}_{P C}$ bound in the previous section for $Z=X_{1}$ and $Z=X_{2}$, it was not necessary to specify the distribution which achieves the bound, since it was an outer bound. On the other hand, when evaluating the Cover-Leung bound, since it is an achievability, it is necessary to give a distribution which achieves the bound.

The dependence balance bounds corresponding to the parallel channel choices $Z=X_{1}$ and $Z=X_{2}$, along with the cut-set upper bound and the Cover-Leung achievable rate region are shown in Figs. 4 and 5. It is interesting to note that our bound improves upon the cut-set bound at all points where the Cover-Leung achievable rate region is strictly larger than the capacity region without feedback. In other words, our bound improves upon the cut-set bound at all points where feedback increases capacity.

We should remark that our choices of parallel channels; namely, $Z=X_{1}$ and $Z=X_{2}$ are the simplest ones which ensure that $I\left(X_{1} ; X_{2} \mid Y, Z, T\right)=0$ but they yield fixed information leaks. We believe that by a more elaborate choice of a parallel channel, i.e., by carefully selecting a parameterized parallel channel $p^{+}\left(z \mid x_{1}, x_{2}, y, t\right)$ such that $I\left(X_{1} ; X_{2} \mid Y, Z, T\right)=0$, one would still be able to restrict the input distributions to a conditionally independent form and then optimize the parameters of the parallel channel to minimize the information leak terms. This approach can potentially improve upon our outer bound. However, for explicitly characterizing such outer bounds, one might require a new approach and possibly a different composite function than the one used in this paper.

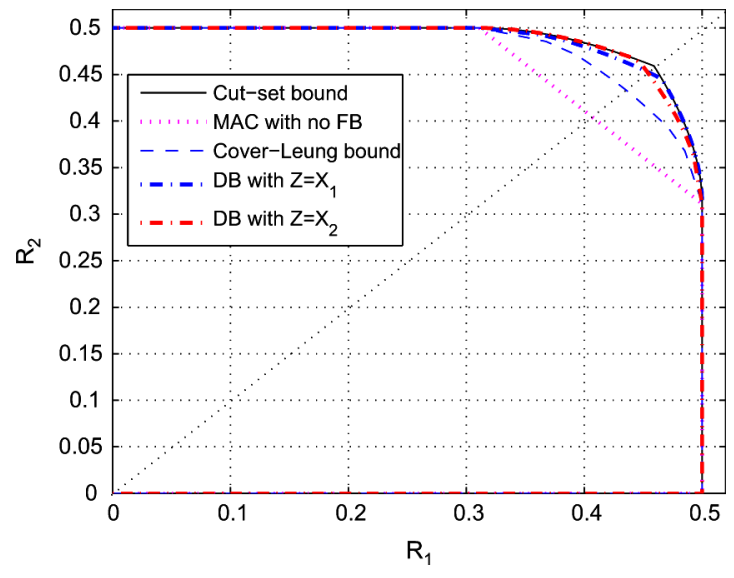

Fig. 4. Illustration of our bounds for the capacity of binary additive noisy MAC-FB.

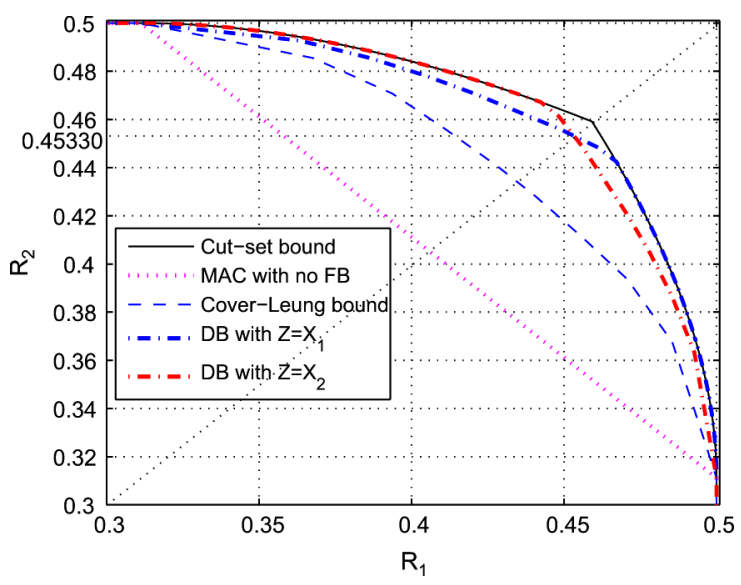

Fig. 5. An enlarged illustration of the portion of Fig. 4 where feedback increases capacity.

\section{The Capacity Region of the Binary ERASURe MAC-FB}

The capacity region of a class of discrete memoryless MAC-FB was characterized in [8] by establishing a converse and it was shown to be equal to the Cover-Leung achievable rate region. This class of channels satisfy the property that at least one of the channel inputs, say $X_{1}$, can be written as a deterministic function of the other channel input $X_{2}$ and the channel output $Y$. The binary erasure MAC, where $Y=X_{1}+X_{2}$, falls into this class of channels. In addition, the binary erasure MAC-FB is the noiseless version of the binary additive noisy MAC-FB studied in this paper.

Willems showed in [12] that a binary selection of auxiliary random variable is sufficient to obtain the sum-rate point of the capacity region of the binary erasure MAC-FB. In this section, we will show that by using our results for composite functions which were presented in previous sections, it is possible to obtain all points on the boundary of this capacity region using a binary auxiliary random variable. The feedback capacity region of this channel is given by the CoveLeung achievable rate region 
given in (143)-(145) which can be simplified for the binary erasure MAC-FB as

$$
\begin{aligned}
R_{1} & \leq H\left(X_{1} \mid T\right) \\
R_{2} & \leq H\left(X_{2} \mid T\right) \\
R_{1}+R_{2} & \leq H(Y) .
\end{aligned}
$$

We now state the main result of this section.

Theorem 3: The feedback capacity region of the binary erasure MAC is given as

$$
\begin{aligned}
\mathcal{C}=\bigcup_{\left(u_{1}, u_{2}\right) \in \mathcal{S}}\left\{\left(R_{1}, R_{2}\right):\right. & R_{1} \leq h\left(\phi\left(2 u_{1}\right)\right) \\
& R_{2} \leq h\left(\phi\left(2 u_{2}\right)\right) \\
R_{1}+R_{2} \leq h\left(f\left(2 u_{1}, 2 u_{2}\right)\right)+1 & \left.\quad-f\left(2 u_{1}, 2 u_{2}\right)\right\}
\end{aligned}
$$

where the set $\mathcal{S}$ is defined as

$$
\mathcal{S}=\left\{\left(u_{1}, u_{2}\right): 0 \leq u_{1} \leq \frac{1}{4} ; 0 \leq u_{2} \leq \frac{1}{4}\right\} .
$$

Proof: We start by obtaining three upper bounds on the expressions appearing in the bounds (162)-(164). We first have

$$
H\left(X_{1} \mid T\right) \leq h\left(\phi\left(2 u_{1}\right)\right) .
$$

Similarly, we also have

$$
H\left(X_{2} \mid T\right) \leq h\left(\phi\left(2 u_{2}\right)\right) .
$$

We now obtain an upper bound on $H(Y)$, by first noting that

$$
H(Y)=h^{(3)}\left(P_{Y}(0), P_{Y}(1), P_{Y}(2)\right)
$$

where

$$
\begin{aligned}
& P_{Y}(0)=\sum_{t} p_{t} q_{1 t} q_{2 t} \\
& P_{Y}(1)=\sum_{t} p_{t}\left(q_{1 t}+q_{2 t}-2 q_{1 t} q_{2 t}\right) \\
& P_{Y}(2)=\sum_{t} p_{t}\left(1-q_{1 t}\right)\left(1-q_{2 t}\right) .
\end{aligned}
$$

Now, we use the following inequality established in [12]:

$$
\begin{aligned}
h^{(3)}(a, b, c) & =\frac{1}{2} h^{(3)}(a, b, c)+\frac{1}{2} h^{(3)}(c, b, a) \\
& \leq h^{(3)}\left(\frac{a+c}{2}, b, \frac{a+c}{2}\right) \\
& =h(b)+1-b
\end{aligned}
$$

where (174) follows by the concavity of the entropy function and by the application of Jensen's inequality [14]. Using (175) and continuing from (169), we obtain

$$
\begin{aligned}
H(Y) & =h^{(3)}\left(P_{Y}(0), P_{Y}(1), P_{Y}(2)\right) \\
& \leq h\left(P_{Y}(1)\right)+1-P_{Y}(1)
\end{aligned}
$$

$$
=h(u)+1-u
$$

where $u$ is defined in (53). Using (167), (168), and (178), we can write an outer bound $\mathcal{O}_{1}$ on the capacity region as follows:

$$
\mathcal{O}_{1}=\bigcup_{\left(u_{1}, u_{2}, u\right) \in \mathcal{P}} \mathcal{O}_{1}\left(u_{1}, u_{2}, u\right)
$$

where

$$
\begin{aligned}
\mathcal{O}_{1}\left(u_{1}, u_{2}, u\right)=\left\{\left(R_{1}, R_{2}\right)\right. & : R_{1} \leq h\left(\phi\left(2 u_{1}\right)\right) \\
& R_{2} \leq h\left(\phi\left(2 u_{2}\right)\right) \\
& \left.R_{1}+R_{2} \leq h(u)+1-u\right\}
\end{aligned}
$$

and the set $\mathcal{P}$ is defined in (86). We will now obtain a simpler characterization of $\mathcal{O}_{1}$ in terms of two variables $\left(u_{1}, u_{2}\right)$ by showing that $\mathcal{O}_{1} \equiv \mathcal{O}_{2}$, where

$$
\mathcal{O}_{2}=\bigcup_{\left(u_{1}, u_{2}\right) \in \mathcal{S}} \mathcal{O}_{2}\left(u_{1}, u_{2}\right)
$$

where

$$
\begin{aligned}
\mathcal{O}_{2}\left(u_{1}, u_{2}\right)=\left\{\left(R_{1}, R_{2}\right):\right. & R_{1} \leq h\left(\phi\left(2 u_{1}\right)\right) \\
& R_{2} \leq h\left(\phi\left(2 u_{2}\right)\right) \\
R_{1} & +R_{2} \leq h\left(f\left(2 u_{1}, 2 u_{2}\right)\right)+1 \\
& \left.\quad-f\left(2 u_{1}, 2 u_{2}\right)\right\} .(182)
\end{aligned}
$$

The proof of the claim $\mathcal{O}_{1} \equiv \mathcal{O}_{2}$ is given in the Appendix . Hence, we have an outer bound on the capacity region as given by $\mathrm{O}_{2}$.

The outer bound $\mathcal{O}_{2}$ is evaluated over the set of pairs $\left(u_{1}, u_{2}\right)$ such that $u_{1}, u_{2} \in\left[0, \frac{1}{4}\right]$. For any such arbitrary pair $\left(u_{1}, u_{2}\right)$, an input distribution which achieves the set of rate pairs specified by $\mathcal{O}_{2}\left(u_{1}, u_{2}\right)$ is obtained by selecting $|\mathcal{T}|=2$, and

$$
\begin{aligned}
p_{0} & =p_{1}=\frac{1}{2} \\
q_{10} & =1-q_{11}=\phi\left(2 u_{1}\right) \\
q_{20} & =1-q_{21}=\phi\left(2 u_{2}\right) .
\end{aligned}
$$

The set of rates achievable by the distribution specified in (183)-(185) are obtained as

$$
\begin{aligned}
R_{1} & \leq H\left(X_{1} \mid T\right)=h\left(\phi\left(2 u_{1}\right)\right) \\
R_{2} & \leq H\left(X_{2} \mid T\right)=h\left(\phi\left(2 u_{2}\right)\right) \\
R_{1}+R_{2} & \leq H(Y)=h\left(f\left(2 u_{1}, 2 u_{2}\right)\right)+1-f\left(2 u_{1}, 2 u_{2}\right) .
\end{aligned}
$$

This shows that the capacity region of binary erasure MAC-FB can be obtained by a binary and uniform selection of the auxiliary random variable $T$.

The capacity region of the binary erasure MAC with and without feedback and the cut-set bound are illustrated in Figs. 6 and 7. It was shown in [12] that the sum-rate point on the boundary of the capacity region lies strictly below the "total cooperation" line. This is equivalent to saying that the cut-set bound is not tight for the sum-rate point. From our result, it is 


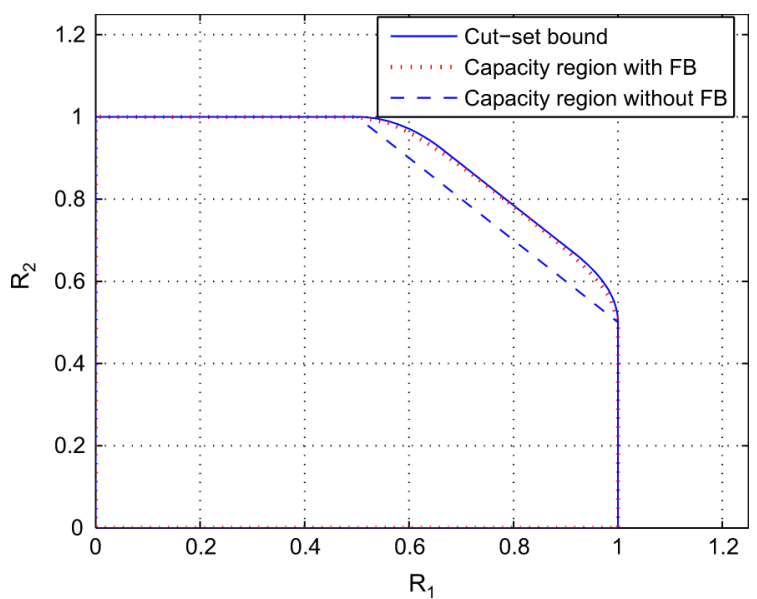

Fig. 6. Illustration of the capacity region of binary erasure MAC-FB.

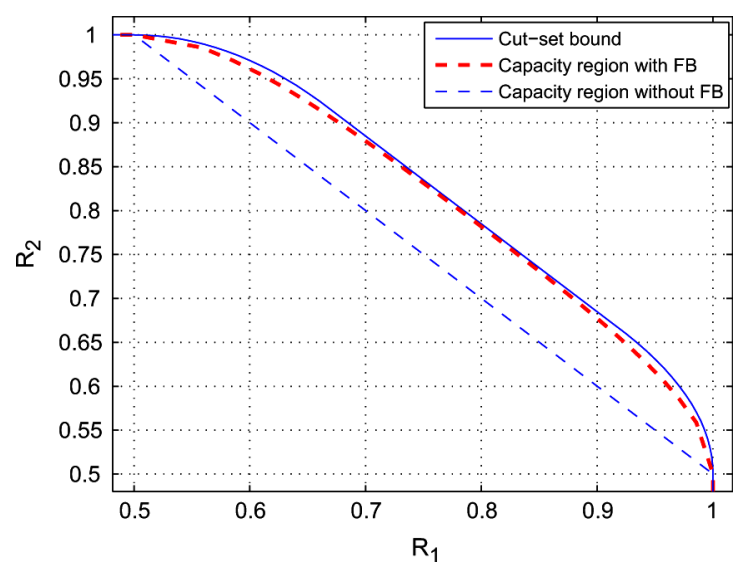

Fig. 7. An enlarged illustration of the portion of Fig. 6 where feedback increases capacity.

now clear that the cut-set bound is not tight for asymmetric rate pairs either. In fact, it is not tight at all boundary points where feedback increases capacity.

Moreover, our result also shows that a simple selection of binary and uniform $T$ is sufficient to evaluate the boundary of the capacity region of binary erasure MAC-FB. Simple feedback strategies for a class of two-user MAC-FB were developed in [13]. It was shown that for the binary erasure MAC, these feedback strategies yield all rate points for a binary selection of the auxiliary random variable $T$. Thus, our result shows that these feedback strategies are indeed optimal for the binary erasure MAC-FB and yield all rates on the boundary of its feedback capacity region.

\section{CONCLUSION}

In this paper, we obtained a new outer bound on the capacity region of a MAC-FB by using the idea of dependence balance. We considered a binary additive noisy MAC-FB for which it is known that feedback increases capacity but the feedback capacity region is not known. The best known outer bound on the feedback capacity region of this channel was the cut-set bound. We used the dependence balance bound to improve upon the cut-set bound at all points in the capacity region of this channel where feedback increases capacity. Our result is somewhat surprising once it is realized that the channel we considered in this paper is the discrete version of the two-user Gaussian MAC-FB considered by Ozarow in [3], where the cut-set bound was shown to be tight.

Our outer bound is difficult to evaluate due to an involved auxiliary random variable $T$. For binary inputs, the cardinality bound on $T$ is $|\mathcal{T}| \leq 7$ which makes it intractable to evaluate the outer bound. We overcome this difficulty by making use of composite functions and their properties to obtain a simple characterization of our bound. As an application of the properties of the composite functions developed in this paper, we are also able to completely characterize the Cover-Leung achievable rate region for this channel.

The capacity region of the binary erasure MAC-FB is known and it coincides with the Cover-Leung achievable rate region. Although the capacity region is known in principle, it is not known how to compute the entire region, the difficulty arising again due to the involved auxiliary random variable. We again make use of the composite functions to give an alternate characterization of the capacity region of the binary erasure MAC-FB. In addition, we go on to show that a binary and uniform auxiliary random variable selection is sufficient to evaluate its feedback capacity region.

\section{APPENDIX}

\section{A. Proof of Lemma 1}

For a given distribution $p(t) p\left(x_{1} \mid t\right) p\left(x_{2} \mid t\right)$, we have

$$
\begin{aligned}
H\left(X_{1} \mid Y, X_{2}, T\right)= & \sum_{\left(y, x_{2}, t\right)} \operatorname{Pr}\left(Y=y, X_{2}=x_{2}, T=t\right) \\
& \cdot H\left(X_{1} \mid Y=y, X_{2}=x_{2}, T=t\right) \\
= & \sum_{t}\left[\operatorname{Pr}\left(Y=1, X_{2}=0, T=t\right)\right. \\
& \cdot H\left(X_{1} \mid Y=1, X_{2}=0, T=t\right) \\
& +\operatorname{Pr}\left(Y=2, X_{2}=1, T=t\right) \\
& \left.\cdot H\left(X_{1} \mid Y=2, X_{2}=1, T=t\right)\right] \\
= & \sum_{t} \frac{1}{2}\left[\operatorname{Pr}\left(X_{2}=0, T=t\right) H\left(X_{1} \mid T=t\right)\right. \\
& \left.+\operatorname{Pr}\left(X_{2}=1, T=t\right) H\left(X_{1} \mid T=t\right)\right] \\
= & \frac{1}{2} H\left(X_{1} \mid T\right)
\end{aligned}
$$

where (190) follows from the fact that $X_{1}$ is uniquely determined when we have $Y=0$ or $Y=3$, or we have $\left(Y, X_{2}\right)=$ $(1,1)$ or $\left(Y, X_{2}\right)=(2,0)$ and (191) follows by noting that

$$
\begin{aligned}
& \operatorname{Pr}\left(Y=1, X_{2}=0, T=t\right)=\frac{1}{2} \operatorname{Pr}\left(X_{2}=0, T=t\right) \\
& \operatorname{Pr}\left(Y=2, X_{2}=1, T=t\right)=\frac{1}{2} \operatorname{Pr}\left(X_{2}=1, T=t\right)
\end{aligned}
$$

and

$$
\operatorname{Pr}\left(X_{1}=0 \mid Y=1, X_{2}=0, T=t\right)=\operatorname{Pr}\left(X_{1}=0 \mid T=t\right)
$$

$$
\operatorname{Pr}\left(X_{1}=0 \mid Y=2, X_{2}=1, T=t\right)=\operatorname{Pr}\left(X_{1}=0 \mid T=t\right) .
$$


We have therefore proved (29) and the proof of (30) follows similarly. This completes the proof of Lemma 1.

\section{B. Proof of Lemma 2}

We prove Lemma 2 by considering all four possible cases.

1) If $s_{1} \in\left[0, \frac{1}{2}\right], s_{2} \in\left[0, \frac{1}{2}\right]$, then from (42), $s_{1}=\phi\left(2 s_{1}(1-\right.$ $\left.\left.s_{1}\right)\right), s_{2}=\phi\left(2 s_{2}\left(1-s_{2}\right)\right)$, and hence

$$
v=f\left(2 s_{1}\left(1-s_{1}\right), 2 s_{2}\left(1-s_{2}\right)\right) .
$$

2) If $s_{1} \in\left[\frac{1}{2}, 1\right], s_{2} \in\left[\frac{1}{2}, 1\right]$, then from (42), $s_{1}=1-$ $\phi\left(2 s_{1}\left(1-s_{1}\right)\right), s_{2}=1-\phi\left(2 s_{2}\left(1-s_{2}\right)\right)$, and hence

$$
v=f\left(2 s_{1}\left(1-s_{1}\right), 2 s_{2}\left(1-s_{2}\right)\right) .
$$

3) If $s_{1} \in\left[0, \frac{1}{2}\right], s_{2} \in\left[\frac{1}{2}, 1\right]$, then from (42), $s_{1}=\phi\left(2 s_{1}(1-\right.$ $\left.\left.s_{1}\right)\right), s_{2}=1-\phi\left(2 s_{2}\left(1-s_{2}\right)\right)$, and hence

$$
\begin{aligned}
v=1-f\left(2 s_{1}\right. & \left.\left(1-s_{1}\right), 2 s_{2}\left(1-s_{2}\right)\right) \\
& \stackrel{(\text { a) }}{\geq} f\left(2 s_{1}\left(1-s_{1}\right), 2 s_{2}\left(1-s_{2}\right)\right)
\end{aligned}
$$

where (a) follows by the fact that $f\left(2 s_{1}\left(1-s_{1}\right)\right.$, $\left.2 s_{2}\left(1-s_{2}\right)\right) \leq \frac{1}{2}$.

4) If $s_{1} \in\left[\frac{1}{2}, 1\right], s_{2} \in\left[0, \frac{1}{2}\right]$, then from (42), $s_{1}=1-$ $\phi\left(2 s_{1}\left(1-s_{1}\right)\right), s_{2}=\phi\left(2 s_{2}\left(1-s_{2}\right)\right)$, and hence

$$
\begin{aligned}
v=1-f\left(2 s_{1}\right. & \left.\left(1-s_{1}\right), 2 s_{2}\left(1-s_{2}\right)\right) \\
& \stackrel{(\mathrm{b})}{\geq} f\left(2 s_{1}\left(1-s_{1}\right), 2 s_{2}\left(1-s_{2}\right)\right)
\end{aligned}
$$

where (b) follows by the fact that $f\left(2 s_{1}\left(1-s_{1}\right)\right.$, $\left.2 s_{2}\left(1-s_{2}\right)\right) \leq \frac{1}{2}$.

Thus, for any pair $\left(s_{1}, s_{2}\right)$, where $s_{1} \in[0,1], s_{2} \in[0,1]$, we have shown that $v \geq f\left(2 s_{1}\left(1-s_{1}\right), 2 s_{2}\left(1-s_{2}\right)\right)$.

\section{Proof of Lemma 3}

A function $f(x, y)$ is jointly convex [16] in $(x, y)$ if for any two pairs, $(x, y)$ and $\left(x^{\prime}, y^{\prime}\right)$, we have $f\left(\alpha x+(1-\alpha) x^{\prime}\right.$, $\left.\alpha y+(1-\alpha) y^{\prime}\right) \leq \alpha f(x, y)+(1-\alpha) f\left(x^{\prime}, y^{\prime}\right)$, for all $\alpha \in[0,1]$.

Showing that the function $f(x, y)$ is jointly convex in $(x, y)$ is equivalent to showing that the Hessian matrix $H$ of $f(x, y)$ is positive semi-definite, which is equivalent to showing that the eigenvalues of $H$ are nonnegative. The Hessian matrix $H$ of $f(x, y)$ is

$$
H=\left(\begin{array}{cc}
\frac{\sqrt{1-2 y}}{2(1-2 x)^{3 / 2}} & \frac{-1}{2 \sqrt{(1-2 x)(1-2 y)}} \\
\frac{-1}{2 \sqrt{(1-2 x)(1-2 y)}} & \frac{\sqrt{1-2 x}}{2(1-2 y)^{3 / 2}}
\end{array}\right) .
$$

The two eigenvalues of $H$ are

$$
\begin{aligned}
& \lambda_{1}=0 \\
& \lambda_{2}=\frac{1}{2}\left(\frac{\sqrt{1-2 y}}{(1-2 x)^{3 / 2}}+\frac{\sqrt{1-2 x}}{(1-2 y)^{3 / 2}}\right)
\end{aligned}
$$

which are nonnegative for all $0 \leq x \leq \frac{1}{2}$ and $0 \leq y \leq \frac{1}{2}$, thus completing the proof.

\section{Proof of Lemma 4}

It suffices to show that for a fixed $u_{2}$, the function $g\left(u_{1}, u_{2}\right)$ is monotonically decreasing in $u_{1}$. Substituting the value of $f\left(2 u_{1}, 2 u_{2}\right)$, we have

$$
\begin{aligned}
g\left(u_{1}, u_{2}\right) & =\frac{1}{2} h\left(\frac{1-\left(\phi\left(2 u_{1}\right)+\phi\left(2 u_{2}\right)-2 \phi\left(2 u_{1}\right) \phi\left(2 u_{2}\right)\right)}{2}\right) \\
& =\frac{1}{2} h\left(\frac{1}{2}-\frac{\phi\left(2 u_{2}\right)}{2}-\frac{\phi\left(2 u_{1}\right)\left(1-2 \phi\left(2 u_{2}\right)\right)}{2}\right) .
\end{aligned}
$$

Now using the fact that $\phi(2 s)$ is increasing in $s$ for $s \in\left[0, \frac{1}{4}\right]$, we have that for $u_{1}^{\prime} \geq u_{1}, \phi\left(2 u_{1}^{\prime}\right) \geq \phi\left(2 u_{1}\right)$. Moreover, the following holds:

$$
\frac{\phi\left(2 u_{1}^{\prime}\right)\left(1-2 \phi\left(2 u_{2}\right)\right)}{2} \geq \frac{\phi\left(2 u_{1}\right)\left(1-2 \phi\left(2 u_{2}\right)\right)}{2}
$$

since $\phi\left(2 u_{2}\right) \leq \frac{1}{2}$. Now using the above inequality along with the fact that the binary entropy function $h(s)$ is increasing for $0 \leq s \leq \frac{1}{2}$, we have that for $u_{1}^{\prime} \geq u_{1}$

$$
\begin{aligned}
\frac{1}{2} h & \left(\frac{1}{2}-\frac{\phi\left(2 u_{2}\right)}{2}-\frac{\phi\left(2 u_{1}\right)\left(1-2 \phi\left(2 u_{2}\right)\right)}{2}\right) \\
& \geq \frac{1}{2} h\left(\frac{1}{2}-\frac{\phi\left(2 u_{2}\right)}{2}-\frac{\phi\left(2 u_{1}^{\prime}\right)\left(1-2 \phi\left(2 u_{2}\right)\right)}{2}\right) .
\end{aligned}
$$

This shows that for a fixed $u_{2}$, the function $g\left(u_{1}, u_{2}\right)$ is monotonically decreasing in $u_{1}$. As the function is symmetric in $u_{1}$ and $u_{2}$, the monotonicity of $g\left(u_{1}, u_{2}\right)$ in $\left(u_{1}, u_{2}\right)$ follows.

To show the concavity of $g\left(u_{1}, u_{2}\right)$ in the pair $\left(u_{1}, u_{2}\right)$, we first note from Lemma 3 that $f\left(2 u_{1}, 2 u_{2}\right)$ is jointly convex in the pair $\left(u_{1}, u_{2}\right)$. We define another function

$$
\xi\left(u_{1}, u_{2}\right)=\frac{1-f\left(2 u_{1}, 2 u_{2}\right)}{2} .
$$

Note that $\xi\left(u_{1}, u_{2}\right)$ is jointly concave in the pair $\left(u_{1}, u_{2}\right)$. Furthermore, the binary entropy function $h(s)$ is concave and nondecreasing for $s \in[0,1 / 2]$. Hence, rewriting the function $g\left(u_{1}, u_{2}\right)$ as a composition of two functions, we obtain

$$
g\left(u_{1}, u_{2}\right)=\frac{1}{2} h\left(\xi\left(u_{1}, u_{2}\right)\right) .
$$

From the theory of composite functions [16], we know that a composite function $f_{1}\left(f_{2}(s)\right)$ is concave in $s$ if $f_{1}(\cdot)$ is concave and nondecreasing and $f_{2}(s)$ is concave in $s$. Identifying $f_{1}(\cdot)$ with $h(\cdot)$ and $f_{2}\left(u_{1}, u_{2}\right)$ with $\xi\left(u_{1}, u_{2}\right)$, the concavity of $g\left(u_{1}, u_{2}\right)$ in the pair $\left(u_{1}, u_{2}\right)$ is established.

\section{E. Proof of the Claim $\mathcal{O}_{1} \equiv \mathcal{O}_{2}$}

The inclusion $\mathcal{O}_{2} \subseteq \mathcal{O}_{1}$ is straightforward by forcing $u=$ $f\left(2 u_{1}, 2 u_{2}\right)$ in $\mathcal{O}_{1}$. We will now show that $\mathcal{O}_{1} \subseteq \mathcal{O}_{2}$. For this purpose, we will need the following lemma.

Lemma 5: The function

$$
\mu(s)=h(s)+1-s
$$


is concave in $s$ for $s \in[0,1]$ and takes its maximum value at $s=\frac{1}{3}$. Moreover, the function $\mu(s)$ is increasing in $s$ for $s \in\left[0, \frac{1}{3}\right]$ and decreasing in $s$ for $s \in\left[\frac{1}{3}, 1\right]$.

The proof of this lemma follows from the fact that both $h(s)$ and $-s$ are concave in $s$.

Now consider any arbitrary triple $\left(u_{1}, u_{2}, u\right) \in \mathcal{P}$. We can classify any such triple into one of the following cases.

1) If $f\left(2 u_{1}, 2 u_{2}\right) \leq u \leq \frac{1}{2}$ : for any $\operatorname{such}\left(u_{1}, u_{2}, u\right)$, there exists a pair $\left(\bar{u}_{1}, \bar{u}_{2}\right)$, such that

$$
\begin{aligned}
u_{1} & \leq \bar{u}_{1} \leq \frac{1}{4} \\
u_{2} & \leq \bar{u}_{2} \leq \frac{1}{4} \\
u & =f\left(2 \bar{u}_{1}, 2 \bar{u}_{2}\right) .
\end{aligned}
$$

One such pair $\left(\bar{u}_{1}, \bar{u}_{2}\right)$ can be obtained as follows. Using the fact that for a fixed $u_{1}, f\left(2 u_{1}, 2 u_{2}\right)$ is increasing in $u_{2}$, we select $\bar{u}_{1}=u_{1}$ and solve for $u_{2} \leq \bar{u}_{2} \leq \frac{1}{4}$ for which $f\left(2 \bar{u}_{1}, 2 \bar{u}_{2}\right)=u$. The required $\bar{u}_{2}$ is obtained as

$$
\bar{u}_{2}=\frac{1}{4}\left(1-\frac{(1-2 u)^{2}}{\left(1-4 u_{1}\right)}\right) .
$$

For such a pair $\left(\bar{u}_{1}, \bar{u}_{2}\right)$, the following inequalities hold:

$$
\begin{aligned}
h\left(\phi\left(2 u_{1}\right)\right) & =h\left(\phi\left(2 \bar{u}_{1}\right)\right) \\
h\left(\phi\left(2 u_{2}\right)\right) & \leq h\left(\phi\left(2 \bar{u}_{2}\right)\right) \\
h(u)+1-u & =h\left(f\left(2 \bar{u}_{1}, 2 \bar{u}_{2}\right)\right)+1-f\left(2 \bar{u}_{1}, 2 \bar{u}_{2}\right) .
\end{aligned}
$$

2) If $f\left(2 u_{1}, 2 u_{2}\right) \leq \frac{1}{2} \leq u \leq 1-\left(u_{1}+u_{2}\right)$, then we have by Lemma 5

$$
\begin{aligned}
h(u)+1-u & \leq h\left(\frac{1}{2}\right)+1-\frac{1}{2} \\
& =\frac{3}{2} .
\end{aligned}
$$

Now consider the pair $\left(\bar{u}_{1}, \bar{u}_{2}\right)=\left(\frac{1}{4}, \frac{1}{4}\right)$, for which we have $f\left(2 \bar{u}_{1}, 2 \bar{u}_{2}\right)=\frac{1}{2}$. Hence, we have that

$$
\begin{aligned}
h\left(\phi\left(2 u_{1}\right)\right) & \leq h\left(\phi\left(2 \bar{u}_{1}\right)\right)=1 \\
h\left(\phi\left(2 u_{2}\right)\right) & \leq h\left(\phi\left(2 \bar{u}_{2}\right)\right)=1 \\
h(u)+1-u & \leq h\left(f\left(2 \bar{u}_{1}, 2 \bar{u}_{2}\right)\right)+1-f\left(2 \bar{u}_{1}, 2 \bar{u}_{2}\right)=\frac{3}{2} .
\end{aligned}
$$

We have thus shown that for any triple $\left(u_{1}, u_{2}, u\right)$, there exists a pair $\left(\bar{u}_{1}, \bar{u}_{2}\right)$, such that $\mathcal{O}_{1}\left(u_{1}, u_{2}, u\right) \subseteq \mathcal{O}_{2}\left(\bar{u}_{1}, \bar{u}_{2}\right)$, which in turn implies that $\mathcal{O}_{1} \subseteq \mathcal{O}_{2}$, and consequently $\mathcal{O}_{1} \equiv \mathcal{O}_{2}$.

\section{REFERENCES}

[1] R. Tandon and S. Ulukus, "A new upper bound for a binary additive noisy multiple access channel with feedback," in Proc. IEEE Global Communications Conf., New Orleans, LA, Nov. 2008.

[2] N. Gaarder and J. Wolf, "The capacity region of a multiple-access discrete memoryless channel can increase with feedback," IEEE Trans. Inf. Theory, vol. IT-21, no. 1, pp. 100-102, Jan. 1975.
[3] L. Ozarow, "The capacity of the white Gaussian multiple access channel with feedback," IEEE Trans. Inf. Theory, vol. IT-30, no. 4 pp. 623-629, Jul. 1984

[4] J.P. M. Schalkwijk and T. Kailath, "A coding scheme for additive noise channels with feedback-Part I: No bandwidth constraint," IEEE Trans. Inf. Theory, vol. IT-12, no. 2, pp. 172-182, Apr. 1966.

[5] T. M. Cover and C. S. K. Leung, "An achievable rate region for the multiple access channel with feedback," IEEE Trans. Inf. Theory, vol. IT-27, no. 3, pp. 292-298, May 1981.

[6] G. Kramer, "Directed Information for Channels with Feedback," Ph.D. dissertation, ETH Zurich, Zurich, Switzerland, 1998.

[7] S. I. Bross and A. Lapidoth, "An improved achievable rate region for the discrete memoryless two-user multiple-access channel with noiseless feedback," IEEE Trans. Inf. Theory, vol. 51, no. 3, pp. 811-833, Mar. 2005.

[8] F. M. J. Willems, "The feedback capacity region of a class of discrete memoryless multiple access channels," IEEE Trans. Inf. Theory, vol. IT-28, no. 1, pp. 93-95, Jan. 1982.

[9] A. P. Hekstra and F. M. J. Willems, "Dependence balance bounds for single output two-way channels," IEEE Trans. Inf. Theory, vol. 35, no. 1, pp. 44-53, Jan. 1989.

[10] G. Kramer, "Capacity results for the discrete memoryless network," IEEE Trans. Inf. Theory, vol. 49, no. 1, pp. 4-21, Jan. 2003.

[11] A. J. Vinck, W. L. M. Hoeks, and K. A. Post, "On the capacity of the two-user $M$-ary multiple-access channel with feedback," IEEE Trans. Inf. Theory, vol. IT-31, no. 4, pp. 540-543, Jul. 1985.

[12] F. Willems, "On multiple access channels with feedback," IEEE Trans. Inf. Theory, vol. IT-30, no. 6, pp. 842-845, Nov. 1984.

[13] G. Kramer, "Feedback strategies for a class of two-user multiple access channels," IEEE Trans. Inf. Theory, vol. 45, no. 6, pp. 2054-2059, Sep. 1999.

[14] T. M. Cover and J. A. Thomas, Elements of Information Theory. New York: Wiley, 1991.

[15] Z. Zhang, T. Berger, and J. P. M. Schalkwijk, "New outer bounds to capacity regions of two-way channels," IEEE Trans. Inf. Theory, vol. IT-32, no. 3, pp. 383-386, May 1986.

[16] S. Boyd and L. Vandenberghe, Convex Optimization. Cambridge, U.K.: Cambridge Univ. Press, 2004.

Ravi Tandon (S'03) received the B.Tech. degree in electrical engineering from the Indian Institue of Technology, Kanpur, India, in 2004.

$\mathrm{He}$ is currently working towards the Ph.D. degree in the Department of Electrical and Computer Engineering, University of Maryland, College Park. His research interests include network information theory and wireless communication theory.

Sennur Ulukus (S'90-M'98) received the B.S. and M.S. degrees in electrical and electronics engineering from Bilkent University, Ankara, Turkey, in 1991 and 1993, respectively, and the Ph.D. degree in electrical and computer engineering from Rutgers University, New Brunswicj, NJ, in 1998.

During her Ph.D. studies, she was with the Wireless Information Network Laboratory (WINLAB), Rutgers University. From 1998 to 2001, she was a Senior Technical Staff Member at AT\& T Labs-Research, Florham Park NJ. In 2001, she joined the University of Maryland, College Park, where she is currently an Associate Professor in the Department of Electrical and Computer Engineering, with a joint appointment at the Institute for Systems Research (ISR). Her research interests are in wireless communication theory and networking, network information theory for wireless networks, signal processing for wireless communications and security for multiuser wireless communications.

Prof. Ulukus is a recipient of the 2005 NSF CAREER Award, and a corecipient of the 2003 IEEE Marconi Prize Paper Award in Wireless Communications. She serves/served as an Associate Editor for the IEEE TRANSACTIONS ON INFORMATION THEORY since 2007, as an Associate Editor for the IEEE TRANSACTIONS ON COMMUNICATIONS between 2003 and 2007, as a Guest Editor for the IEEE JOURNAL ON SELECTED AREAS IN COMMUNICATIONS in 2006-2008, as the Co-Chair of the Communication Theory Symposium at the 2007 IEEE Global Telecommunications Conference, as the Co-Chair of the Medium Access Control (MAC) Track at the 2008 IEEE Wireless Communications and Networking Conference, as the Co-Chair of the Wireless Communications Symposium at the 2010 IEEE International Conference on Communications, and as the Secretary of the IEEE Communication Theory Technical Committee (CTTC) in 2007-2009. 